\title{
Measurements of Turbulent Convection Speeds in Multistream Jets Using Time-Resolved PIV
}

\author{
James Bridges ${ }^{*}$ and Mark P. Wernet ${ }^{\dagger}$ \\ NASA Glenn Research Center, Cleveland, OH 44135
}

\begin{abstract}
Convection speeds of turbulent velocities in jets, including multi-stream jets with and without flight stream, were measured using an innovative application of time-resolved particle image velocimetry. The paper describes the unique instrumentation and data analysis that allows the measurement to be made. Extensive data is shown that relates convection speed, mean velocity, and turbulent velocities for multiple jet cases. These data support the overall observation that the local turbulent convection speed is roughly that of the local mean velocity, biased by the relative intensity of turbulence.
\end{abstract}

\section{Nomenclature}

nozzle area

speed of sound

nozzle diameter

area-equivalent nozzle diameter

Mach number

acoustic Mach number $\left(M a=U_{j} / c_{\infty}\right)$

nozzle total pressure ratio

nozzle total temperature ratio

turbulent kinetic energy

temperature

jet velocity

convection speed

axial coordinate

Subscripts

${ }_{1,2,3} \quad$ core, bypass, tertiary stream

0 centerline

eq area-equivalent

j jet exit

$\infty \quad$ ambient

$<\bullet \quad$ average of $\bullet$

\section{Introduction}

Acoustic analogies, used in most physics-based noise prediction methods, include a term called the Doppler factor, that relates the local convection speed of turbulence to the sound propagated to the far field. In effect, this term describes the coupling of vortical motion to the irrotational acoustic field around the jet. In most prediction codes this convection speed is assumed to be some fraction of the local maximum average velocity, typically 0.60.65 times $\mathrm{U}_{0}$, the local centerline velocity. While this might be a reasonable estimate, the truth is that the convection speed varies across the jet, and when there are multiple streams in the plume it is not clear how to apply this approximation when addressing the coupling between turbulent energy and ambient acoustic waves over the entire shear layer jet plume. Some aeroacoustic models ${ }^{1}$ have chosen to use the mean velocity at local inflection points as an approximation for the convection speed. More recently ${ }^{2}$ the convection speed has been taken to be the mean velocity at the location of peak turbulence at any given axial location. Other noise prediction methods $\mathrm{s}^{3,4}$ base

\footnotetext{
*Acoustics Branch, MS 54-3; AIAA Associate Fellow

† Instrumentation and NDE Branch, MS 77-1; AIAA Associate Fellow.
} 
their source models on the space-time correlation of near-field pressure very near the edge of the jet plume. One key factor in determining how well the wave-packets described by these models couple to the acoustic far field is the convection speed. Reducing the convection speed is predicted to reduce the efficiency of the conversion of turbulent energy to acoustic energy.

Given the interest in describing the jet noise source mechanism as being related to convecting turbulent structures, basic measurements of the convection speed should be vital in the formulation of predictive models. The real test of noise models is not just whether they can predict the noise of a simple single-stream jet, but whether they can predict the noise of practical, multi-stream plumes with non-axisymmetric features. The recent work of Papamoschou and Phong ${ }^{5}$ present one such effort to looking at the effect of convection speeds in multi-stream jets. One key to using a predictive method relying on statistical models of convecting turbulence is the prediction of the convection speed itself.

The major objectives of the test were to (i) obtain convection speed data for cold subsonic single-stream jets for comparison with previously published results, (ii) obtain convection speed data for two- and three-stream axisymmetric jet plumes to understand how multiple shear layers affected these speeds, and (iii) acquire convection speed data for asymmetric jet plumes to see if such asymmetry really resulted in lowered convection speeds.

\section{A. Measurement of convection speed}

Previous work, limited to single-stream jets flows, has found that the convection speed varies from the mean velocity, matching the mean velocity only at roughly the lipline or half-velocity point. Measuring convection speed is typically done by space-time correlations, determining the time delay of the peak correlation between two points in the flow. Previously measurements have been made using hotwire anemometry ${ }^{6}$, which is limited to low speed, cold flows, and in a few instances optical methods ${ }^{7}$. These measurements, reproduced in Figure 1, show that convection speed changes smoothly from $0.85 \mathrm{U}_{0}$ at the centerline to $0.4 \mathrm{U}_{0}$ at the outer edges of the jet plume. The typical value of 0.6 picked for flow modeling corresponds to the convection speed on the high-speed side of the shear layer near the peak turbulence. Note that these measurements were all made within the potential core of the jets.
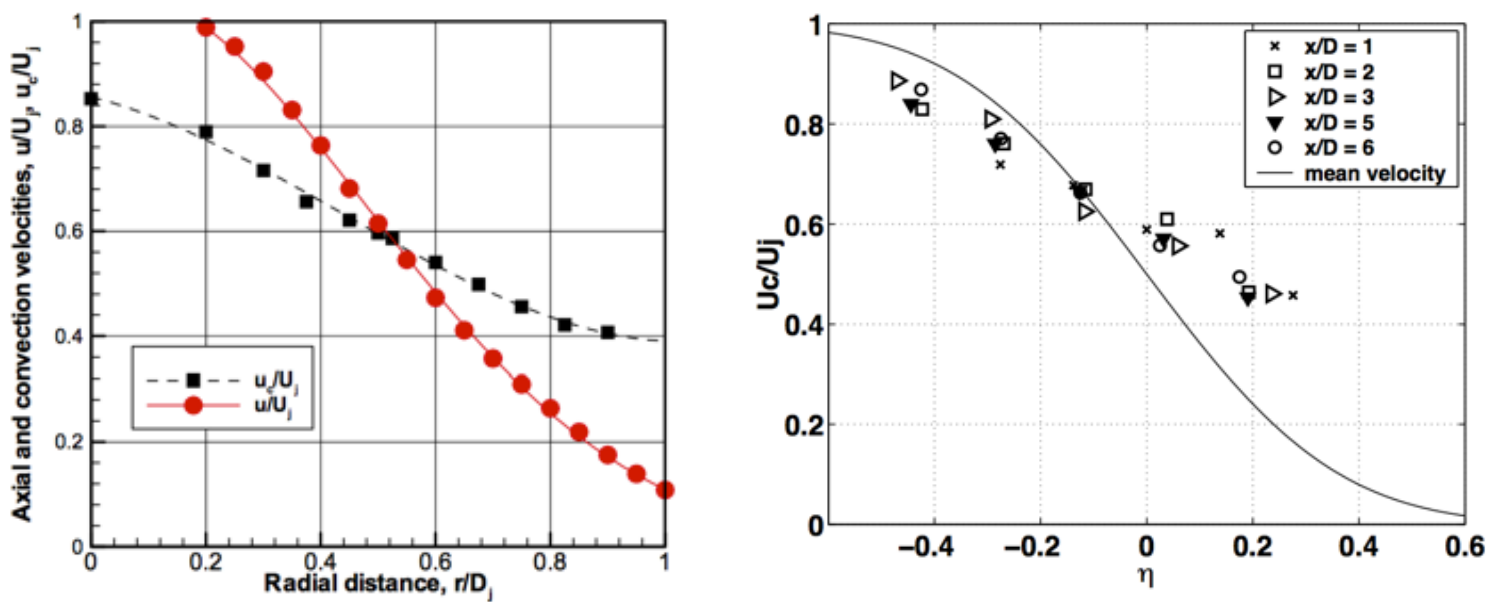

Figure 1 Mean and convection velocity data from previous publications: low speed, cold jet using hotwire, Ref [6] (left) and high speed, cold jet using LDV, Ref [7] (right). Here, $\eta$ is radial displacement from half-velocity point, normalized by jet diameter.

Use of particle image velocimetry (PIV) data for space-time correlations have been infrequent, usually because the implementations were designed to measure spatial fields, and because adequate time resolution is not the method's forte. At heart, the problem is one of information bandwidth, where the product of large images required for PIV cannot be acquired at high sample rates. In 2003 a system was created ${ }^{8}$ using two coupled PIV systems to acquire two velocity fields at variable time delays over the same plane, and from this compute the full space-time correlation of velocity and unsteady Reynolds stress. For its time the test was very advanced, but it was very cumbersome, the data a bit coarse in temporal resolution, and a close study of convection speed was not undertaken. More recently ${ }^{9}$, a high speed pulsed laser was coupled with a high speed, configurable CMOS camera to acquire PIV data at rates up to $25 \mathrm{kHz}$ over a narrow strip of jet flow fields. In this test, conducted on the single-stream jet rig at the NASA Glenn Research Center, convection velocities were determined using space-time correlations on axial 
strips of data roughly $6 \times 0.35$ jet diameters. This provided measurements of convection speed over most of one-half of a jet for many high-speed flow conditions. This system required substantial effort to produce the light sheet, including mounting optics directly downstream in the jet plume. Because the main reason for acquiring this data was to measure the entire space-time correlations of Reynolds stress, measurements of convection speed were only made to better transform the correlations into a stationary frame. In these measurements the convection speed $U_{c}$ did match the mean velocity on the lipline, but was closer to the mean velocity on the centerline than the results shown in Figure 1. The analysis was complicated by the fact that measurements of convection speed in the absence of turbulence (e.g. on the centerline of the potential core) are statistically questionable. A new dataset should address this by measuring $U_{c}$ over the entire jet profile and for many axial stations covering the different domains of jet behavior.

In addition, most measurements of convection speed to date have been made on single-stream jets. However, it is multi-stream jets that are of practical interest, and these have high-speed, high-temperature core flows. It is not clear how convection speed relates to the mean velocities at any given cross-section, where each cross-section has multiple shear layers. Therefore, the objective of the current test was to measure convection speed profiles across multi-stream jet flows at various axial locations to determine an appropriate model for relating convection speed to local mean velocity. As measurements were made on asymmetric multi-stream flows, ideas regarding how the asymmetry changes the far-field noise can also be tested. For this oibjective it is more critical that measurements be made over the entire jet cross-section, and for flows exhibiting as much spatial variation as possible.

\section{B. Prior multi-stream and offset stream nozzle testing}

Recent aeroacoustic testing at the NASA Glenn Research Center Aero-Acoustic Propulsion Lab used a threestream separate flow nozzle system. The axisymmetric model system was made asymmetric using an S-duct outer nozzle and partial blockages of the tertiary stream annulus. Henderson et al. ${ }^{10}$ documented the acoustic far-fields and later ${ }^{11}$ the turbulent flow field for a range of externally mixed three-stream nozzles and a range of high subsonic flows consistent with a variable cycle engine. They found the impact of the third stream varied from inconsequential to significant, as the flow in the third axisymmetric annulus was increased in velocity and mass fraction. Of significant interest was the azimuthal variation in far-field sound produced by making the third stream annulus nonaxisymmetric. Henderson proposed that the impact was largely due to axial vorticity produced in the nonaxisymmetric duct leading to the outer nozzle. Papamoschou explored two-stream nozzles, and more recently threestream nozzles ${ }^{12}$ with non-axisymmetric outer streams and surmised that the change in sound was due to the azimuthal change in convection speed of the large-scale structures (a.k.a. wave packets) produced by differences in the thickness of the outer stream.

The model hardware used in the current paper is the same as that used in Refs. $[10,11,13]$. Flow conditions were picked from the test matrix of the previous work, with the objective of maximizing the shear layer differences and to allow validation against other measurements of acoustics and flow. Some of the flow configurations were also investigated by Ecker et $\mathrm{al}^{14}$ in the same test entry using another novel measuring technique, allowing crossvalidation of methods.

\section{Facility and Model Hardware}

The test was conducted in the NASA Glenn Research Center AeroAcoustic Propulsion Laboratory (AAPL). The AAPL is a $20 \mathrm{~m}$ radius anechoic geodesic hemispherical dome. Acoustic wedges cover the walls of the dome and approximately half of the floor area. The ambient temperature, pressure, and relative humidity were recorded within the dome during the tests. The Nozzle Aeroacoustic Test Rig (NATR) located in the AAPL, is a $1.35 \mathrm{~m}$ diameter free jet that provides the flight stream around the test article. The centerline of the free jet is $3 \mathrm{~m}$ above the floor.

At the downstream end of the NATR is the Dual Flow Jet Exit Rig (DFJER). The DFJER is the structure through which heated air is delivered from the facility compressed air system to the test nozzles. The innermost air stream can be heated using a natural gas combustor to $900 \mathrm{~K}$, with pressure ratios beyond 4 . The second, coannular, air stream can be heated using a heat exchanger up to $420 \mathrm{~K}$ and can also deliver air at pressure ratios beyond 4 . The DFJER was recently modified to accommodate a third stream of air using an additional annular plenum. This third, or tertiary, air stream nominally has the same temperature as the second stream but with independent pressure control.

The rig was instrumented to record total temperature, total pressure at the charging station on both streams. These values, and ambient conditions in the facility, were recorded on the facility computer concurrent with the PIV data acquisition for use in analyzing the data. 


\section{A. Model Hardware}

\section{Configuration definition}

The testing was conducted as part of a larger test program involving the three-stream rig and nozzles. Four nozzle configurations were used in the convection speed portion of the testing.

To produce a single-stream jet, a core nozzle with an internal plug was mounted to the heated stream of the rig, leaving the outer annular streams static, e.g. with no flow. This configuration, shown in

Figure 2, was referred to as ' $2 \mathrm{BBc}$ ', for historical model $2 \mathrm{BB}$, with core only. $2 \mathrm{BBc}$ did have a slight wake in the potential core from the plug, but otherwise provided a single-stream jet with variable temperature to match the flow conditions used in more fundamental tests.

The second objective was met using the axisymmetric three-stream nozzle model, running the outer annulus at the same conditions as the flight stream for the two-stream cases. Nozzle configuration C1T1 was used, having a bypass-to-core area ratio of 2.5 and tip-to-core area ratio of 1 . For the last objective, the C1T1 configuration was modified by using an S-duct to offset the tertiary stream nozzle by $4 \mathrm{~mm}$, a configuration noted as 'SDCT', and modified by blocking off half of the tertiary stream annulus, a configuration noted as 'PART'.

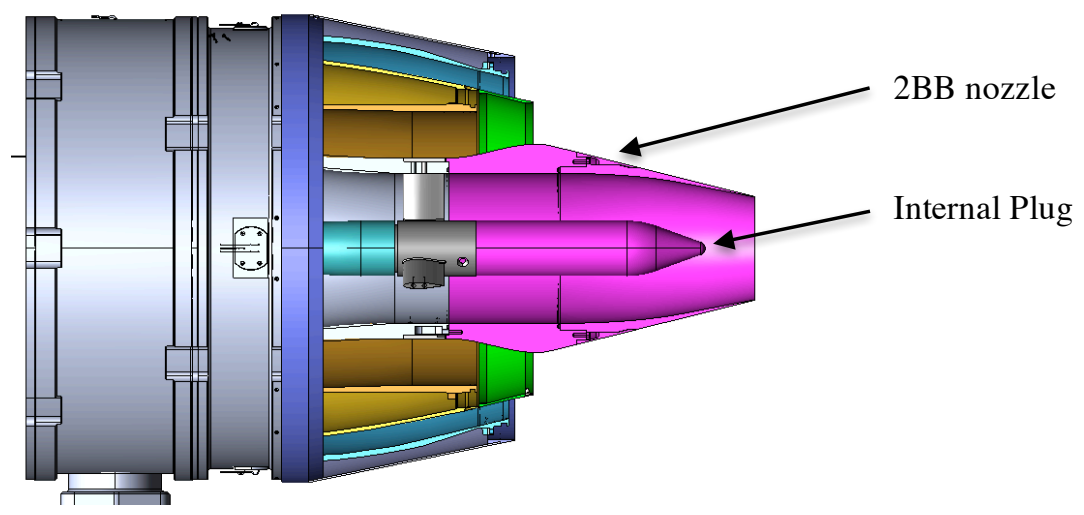

Figure 2 Model configuration 2BBc for single-stream cases. Flow was from inner nozzle only.

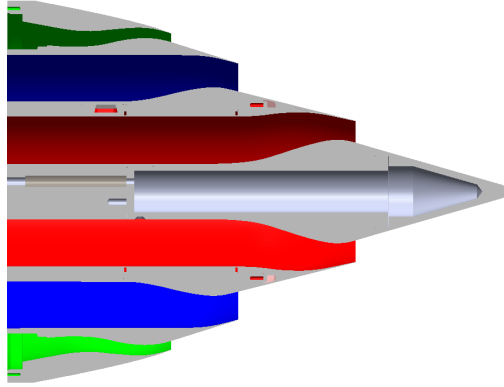

(a)

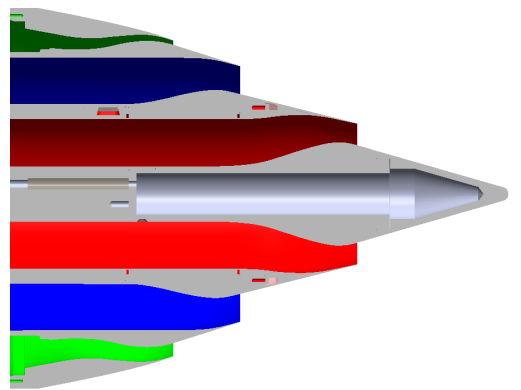

(b)

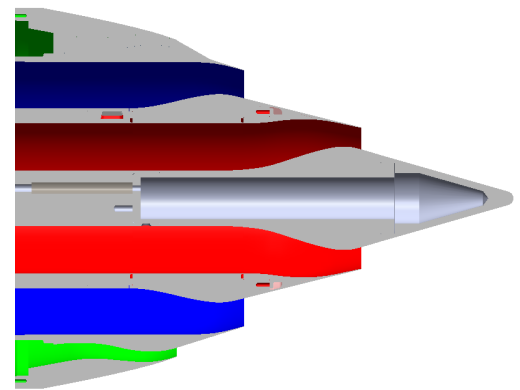

(c)

Figure 3 Model configuration C1T1, used in multi-stream cases. (a) C1T1Axi, (b) C1T1SDCT (with 4mm offset on tertiary stream), (c) CIT1PART (tertiary stream blocked on upper half)

\section{Flow conditions and setpoint definitions}

For the first objective, the single-stream flows given in Table 1 were measured. These flows have all been measured in the NASA Small Hot Jet Acoustic Rig with conventional PIV ${ }^{15}$, and with the exception of setpoint 49 all were measured with time-resolved PIV in a more conventional fashion ${ }^{16}$ as well. These points cover a wide range of acoustic Mach numbers and static temperature ratios as seen in Table 1. 
Table 1 Definitions of Objective 1 setpoints by flow conditions.

\begin{tabular}{|c|ccccc|}
\hline Setpoint & $\begin{array}{c}\text { NPR } \\
\text { Pt/ } P_{\infty}\end{array}$ & $\begin{array}{c}\text { NTR } \\
T t / T_{\infty}\end{array}$ & $\begin{array}{c}\mathrm{V}_{\mathrm{j}} \\
{[\mathrm{m} / \mathrm{s}]}\end{array}$ & $\mathrm{V}_{\mathrm{j}} / \mathrm{C}_{\infty}$ & $\mathrm{T}_{\mathrm{s}, j} / \mathrm{T}_{\infty}$ \\
\hline 3 & 1.197 & 1.000 & 172 & 0.5 & 0.96 \\
\hline 7 & 1.856 & 1.000 & 310 & 0.9 & 0.84 \\
\hline 23 & 1.103 & 1.809 & 172 & 0.5 & 1.76 \\
\hline 27 & 1.361 & 1.918 & 310 & 0.9 & 1.76 \\
\hline 29 & 1.910 & 2.107 & 460 & 1.33 & 1.76 \\
\hline 49 & 1.688 & 3.165 & 500 & 1.48 & 2.70 \\
\hline
\end{tabular}

The flow conditions given in Table 2 were acquired for multi-stream plumes. Because of the limitations of this implementation of time-resolved PIV, convection speeds much above $500 \mathrm{~m} / \mathrm{s}$ would not be measured with much accuracy. Setpoints with $\mathrm{NPR}_{1}=1.8$ at $\mathrm{NTR}_{1}=3$ are technically too high to get more than two time-correlations in the narrow laser sheet, e.g. the time of flight is too long for the observation window set by the laser sheet width. As seen in the table, most of the setpoints pushed this limitation. One extra point was included to test this limit: setpoint 88533. In the tests, convection speed was obtained at this setpoint as well.

Table 2 Matrix of setpoints for Objectives 2 and 3.

\begin{tabular}{|c|cccccccccc|}
\hline Setpoint & $\begin{array}{c}N P R_{1} \\
P t_{1} / P_{\infty}\end{array}$ & $\begin{array}{c}N T R_{1} \\
T_{1} / T_{\infty}\end{array}$ & $\begin{array}{c}\mathrm{NPR}_{2} \\
P t_{2} / P_{\infty}\end{array}$ & $\begin{array}{c}\mathrm{NTR}_{2} \\
\mathrm{Tt}_{2} / T_{\infty}\end{array}$ & $\begin{array}{c}\mathrm{NPR} 3 \\
P t_{3} / P_{\infty}\end{array}$ & $\mathrm{Mf}$ & $\begin{array}{c}\mathrm{V}_{1} \\
{[\mathrm{~m} / \mathrm{s}]}\end{array}$ & $\begin{array}{c}\mathrm{V}_{2} \\
{[\mathrm{~m} / \mathrm{s}]}\end{array}$ & $\begin{array}{c}\mathrm{V}_{3} \\
{[\mathrm{~m} / \mathrm{s}]}\end{array}$ & $\begin{array}{c}\mathrm{V}_{\infty} \\
{[\mathrm{m} / \mathrm{s}]}\end{array}$ \\
\hline 58833 & 1.5 & 3 & 1.8 & 1.25 & 1.8 & 0.3 & 429 & 329 & 329 & 102 \\
\hline 58533 & 1.5 & 3 & 1.8 & 1.25 & 1.5 & 0.3 & 429 & 329 & 276 & 102 \\
\hline 58233 & 1.5 & 3 & 1.8 & 1.25 & 1.2 & 0.3 & 429 & 329 & 188 & 102 \\
\hline 58033 & 1.5 & 3 & 1.8 & 1.25 & 1.06 & 0.3 & 429 & 329 & 102 & 102 \\
\hline 58030 & 1.5 & 3 & 1.8 & 1.25 & 1 & 0 & 429 & 329 & 0 & 0 \\
\hline 55833 & 1.5 & 3 & 1.5 & 1.25 & 1.8 & 0.3 & 429 & 276 & 329 & 102 \\
\hline 85210 & 1.8 & 1.25 & 1.5 & 1.25 & 1.2 & 0 & 334 & 276 & 188 & 0 \\
\hline 88533 & 1.8 & 3 & 1.8 & 1.25 & 1.5 & 0.3 & 510 & 329 & 276 & 102 \\
\hline
\end{tabular}

The multi-stream setpoints were selected for the relative velocities of the different streams, the objective being to have multiple distinct shear layers.

The combinations of model configuration and flow conditions tested are given in Table 3 .

Table 3 Test matrix of selected configurations and conditions.

\begin{tabular}{|c|c|c|c|c|c|c|c|}
\hline Config & Setpoints & & & & & & \\
\hline $\begin{array}{l}\text { 2BBc } \\
\text { C1T1AXI }\end{array}$ & $\begin{array}{c}3 \\
58833\end{array}$ & $\begin{array}{c}7 \\
58033\end{array}$ & $\begin{array}{c}23 \\
58030\end{array}$ & 27 & 29 & 49 & \\
\hline C1T1AXI & & & & 58533 & 55833 & 58233 & 85210 \\
\hline C1T1SDCT & 58833 & & & 58533 & & & 85210 \\
\hline C1T1PART & 58833 & & & 58533 & & & 85210 \\
\hline
\end{tabular}

\section{Instrumentation}

\section{A. Time-Resolved PIV (TRPIV) Implementation Considerations}

To obtain convection speed measurements inside the jet, velocity perturbations must be correlated over space and time. Given discretized measurements of velocity in time, adequate spatial extent must be measured such that turbulence velocity patterns do not convect out of the field of view before successive temporal samples are acquired. This sets a lower limit on the axial extent of the PIV field of view for a given expected convection speed and sample rate. The accuracy of the convection speed measurement is determined by the accuracy of the time-base of the sample, spatial accuracy of the camera, the spatial extent of the field of view (measurements over larger spatial separations are more accurate, but convection speed is not necessarily constant), and the spectral content of the turbulence (short wavelength turbulence produces narrower correlation peaks). Correlating instantaneous velocity fields will produce a spatial correlation function whose shape is relatively well established, and sub-pixel peak- 
finding can be employed in much the same fashion as in PIV image processing to increase the resolution of the measurement.

The application of time-resolved PIV used in this study was unique in that it used a relatively narrow field of view in the streamwise direction in order to maximize the radial domain. Trades between field of view, sample rate, and expected convection speed were analyzed and a reasonable optimization was found with the PIV lasers firing at $25 \mathrm{kHz}$ (velocity samples at $25 \mathrm{kHz}$ ), the camera configured to a $400 \times 1024$ pixel Region of Interest (ROI) mapped to a $55 \mathrm{~mm}$ by $141 \mathrm{~mm}$ field of view $(\mathrm{FoV})$. The main unknown then was the maximum convection speed that could be captured. If the velocity fields were good edge-to-edge, then a $430 \mathrm{~m} / \mathrm{s}$ convection speed could be measured from spatial correlations computed at the two shortest time separations. We wished to maintain multiple time separations for at least two reasons: the image processing used in previous TRPIV often made use of velocity maps before and after in time to remove bad vectors, leaving the measurements not entirely independent; and the uncertainty in velocity can become rather large due to the lack of resolution in x. More details on the image processing are given in Wernet ${ }^{17}$.

Picking locations for measurements was a bit of a guess for the multi-stream jet flows. For the single-stream jet cases, locations were selected for which convection velocities were measured during previous tests, given in Table 4. For the multi-stream cases, we chose to define the plume origin coincident with the fan nozzle exit. The tip of the plug is $258 \mathrm{~mm}$ downstream, making the first measurement station be around $292 \mathrm{~mm}$, avoiding laser flare off the model. Data was acquired at axial locations in Table 5. Given the expected width of the plume, especially by the last axial station, it was expected that three vertical stations would be needed at each axial station, as illustrated by the pink lines in Figure 4. Three vertical passes, centered on the jet centerline and offset top and bottom by $125 \mathrm{~mm}$, provided 145 vertical measurement points over $377 \mathrm{~mm}$ of vertical coverage at a spatial discretization of $2.6 \mathrm{~mm}$ with 4 measurement points overlap on each seam.

In all cases, time-resolved data was acquired at $25 \mathrm{kHz}$ sample rate for 0.15 seconds.

Table 4 Axial locations of acquisition stations for $2 B B c$.

\begin{tabular}{|l|rrrrrr|}
\hline $\mathrm{D}(\mathrm{mm})$ & 95 & & & & & \\
\hline $\mathrm{x} / \mathrm{D}$ & 2 & 3.5 & 6.5 & 9.5 & 12.5 & 15.5 \\
$\mathrm{x}(\mathrm{mm})$ & 190 & 333 & 620 & 905 & 1190 & 1476 \\
\hline
\end{tabular}

Table 5 Axial locations of acquisition stations for multi-stream nozzle, relative to first fan nozzle exit.

\begin{tabular}{|c|c|c|c|c|c|c|c|c|}
\hline $\operatorname{Deq}(\mathrm{mm})$ & 191 & & & & & & & \\
\hline $\mathrm{x} / \mathrm{D}$ & 1.5 & 2 & 3 & 4 & 5 & 6 & 8 & 10 \\
\hline $\mathrm{x}(\mathrm{mm})$ & 286 & 381 & 572 & 762 & 953 & 1143 & 1524 & 1910 \\
\hline
\end{tabular}

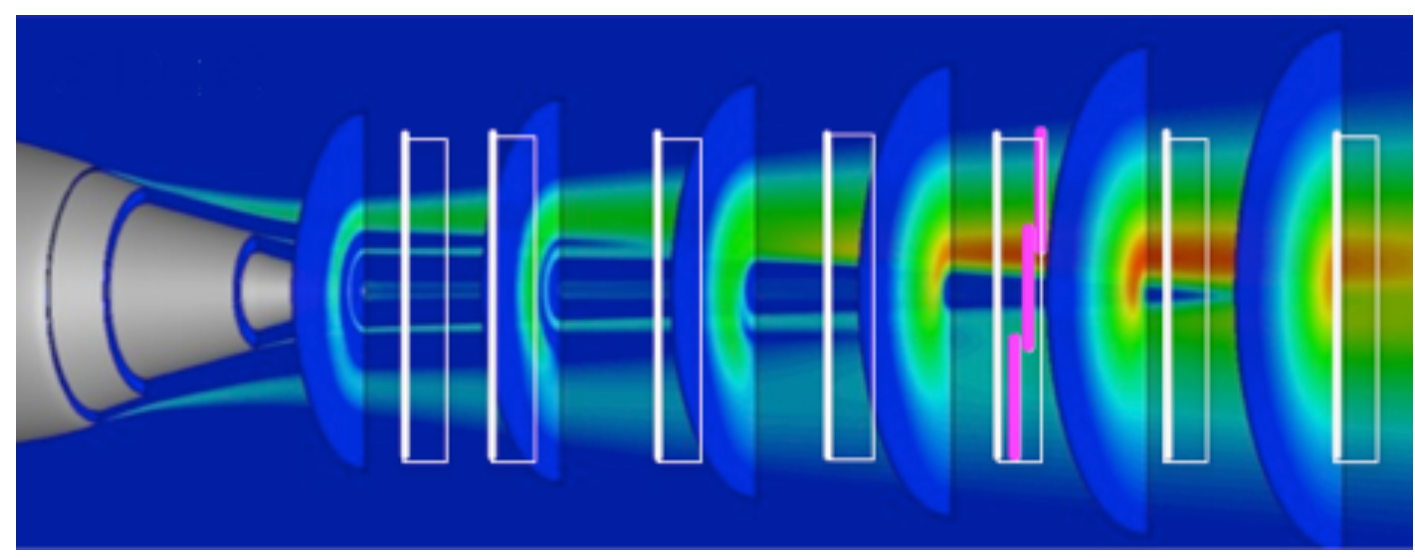

Figure 4 Visual representation of axial measurement locations. White boxes show fields of view acquired on streamwise plane at jet centerline for multistream flows. Pink lines show radial extent of each acquisition.

\section{B. TRPIV Hardware}

The temporally resolved PIV system was implemented using a high repetition rate, dual head laser and a single high-speed camera. A Lee Laser Diode Pumped Solid State (DPSS) laser was used to provide the TR-PIV laser light sheet illumination. Each head in the laser was operated at $25 \mathrm{kHz}$, producing pulse energies of $5 \mathrm{~mJ} / \mathrm{pulse} / \mathrm{head}$. The $1.6 \mathrm{~mm}$ diameter, $\mu^{2}=13$ beam exiting the laser was transformed into a $50 \mathrm{~mm}$ wide by $1 \mathrm{~mm}$ thick light sheet using 
a combination of 4 cylindrical and spherical lenses. A Photron SA-Z CMOS camera operating at $50 \mathrm{kHz}$ was used to record $0.84 \mathrm{sec}$ of TR-PIV image data (41930 frames). The camera was configured with a 400x1024 pixel Region of Interest (ROI), yielding an imaged area of $55 \times 141 \mathrm{~mm}$. A digital delay generator was used to synchronize the camera/laser system timing, which was operated in a "frame-straddling" configuration where image-pairs with a nominal inter-frame time of $1.5 \mu \mathrm{s}$ were recorded at $25 \mathrm{kHz}$. The field of view of the TR-PIV system was insufficient to image the entire diameter of the 3-stream jet flow. Hence, the high-speed camera was mounted on a remotely controlled vertical translation stage enabling acquisition of high-speed image sequences at 3 radial locations across the jet, allowing overlapping fields of view, as shown in Figure 5(a). The entire TR-PIV system was mounted on a large traverse system facilitating acquisition of radial velocity profiles at multiple axial locations downstream from the jet exit. Figure 5(b) shows a photo of the lightsheet positioned just downstream of the $2 \mathrm{BBc}$ nozzle.

The heated flow streams were seeded using a pH stabilized dispersion of $\sim 0.4 \mu \mathrm{m}$ diameter alumina particles in ethanol $^{18}$. The stabilized dispersion of alumina was introduced into the flow well upstream of the model by atomizing the dispersion in each of the three engine streams. The ambient flow from the facility freejet was seeded using a propylene glycol fog of $\sim 0.7 \mu \mathrm{m}$ diameter sized particles. Multiple Roscoe foggers, models 3000 and 6000 , situated in the freejet ejector inlet room of NATR, produce the fog that seed the ambient, or flight, air.

The acquired TR-PIV image data were processed using a multi-pass window offset code, developed in-house. An initial pass using $64 \times 64$ pixels subregions on a $32 \times 32$ pixel grid followed by multiple passes using $32 \times 32$ pixel subregions on a 16x16 pixel grid were used. A final pass using subregion distortion processing completed the data processing.

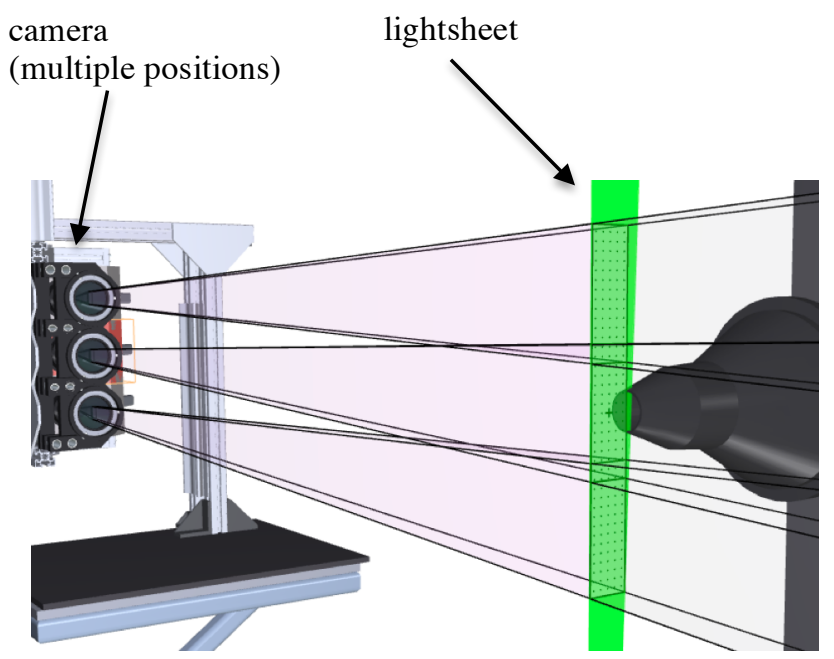

(a)

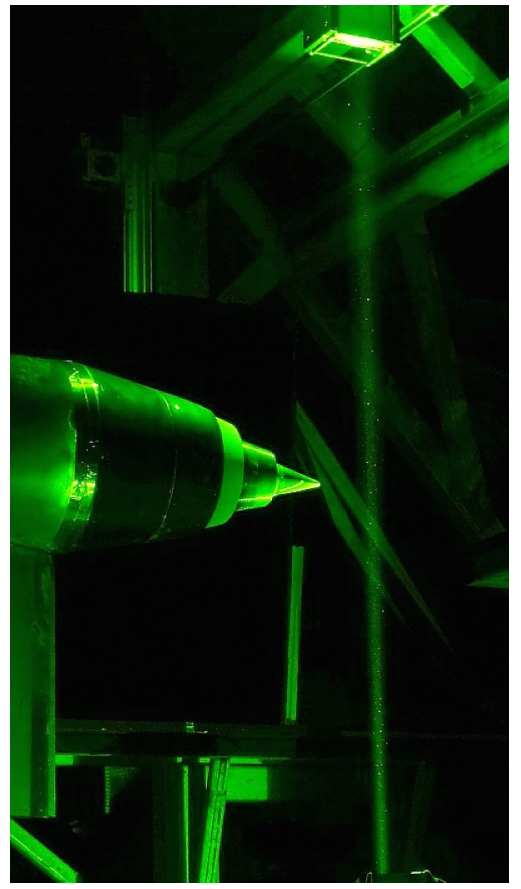

(b)

Figure 5 (a) Illustration of three overlapping fields of view acquired by translating camera to cover the width of the jet. (b) TRPIV operating in NATR with light sheet arranged to give a tall streamwise field of view downstream of nozzle.

\section{Results}

This section is divided into two major sections. The first section provides a quick introduction to the TRPIV data as it was processed into space-time correlations. The second section lays out the extracted convection speeds, along with mean and turbulent velocities, for all the flows measured.

\section{A. Turbulent velocity correlations}

Space-time correlations of the instantaneous axial velocity map records were computed, and by tracing the peak of the correlation in space-time the convection speed of the turbulent velocity was determined. The primary 
constraint on the measurement of convection speed is the short axial extent of the measurement field of view, followed by the constraints of having enough time records to produce a smooth enough correlation to find a statistically significant peak. The former constraint is visually brought home looking at the cross-correlation shown in Figure 7.

Compared with typical PIV measurements there is very little axial extent (roughly 60mm), challenging the estimation of the convection speed, e.g. the slope of the peak correlation in space-time. The line plot in the figure shows the correlation Ruu at the two edges of the window; the difference in time delay tau between peaks and the axial distance between the two slices gives the convection speed $U_{\mathrm{c}}$. Other issues, such as the fact that convection speed changes with displacement (lengthscale-dependent convection speed) cannot be examined with much fidelity with this relatively short spatial domain. To maximize precision of the convection speed, the correlation data, such as those in Figure 7(b), were fitted to a function of the form $A \exp (-\mid \operatorname{tau}-B \mathrm{l} / C)$ over the range of positive $R u u$, and the peak determined from the $B$ coefficient of the fitted curve.

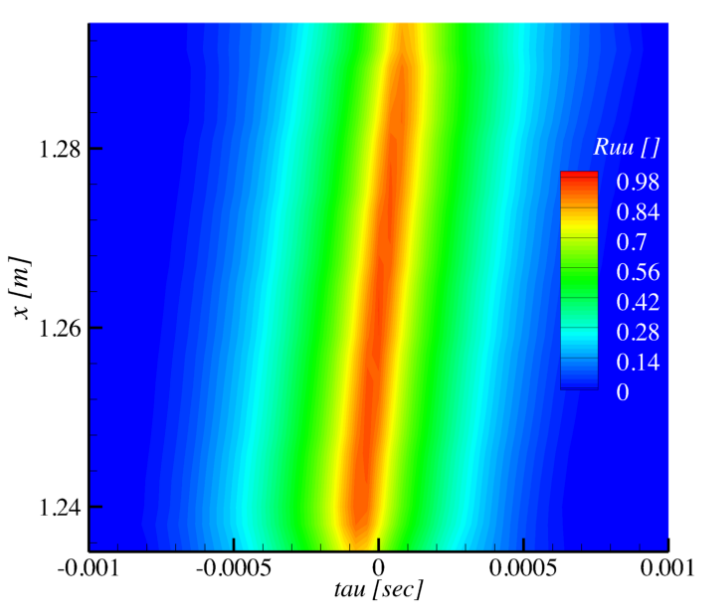

(a)

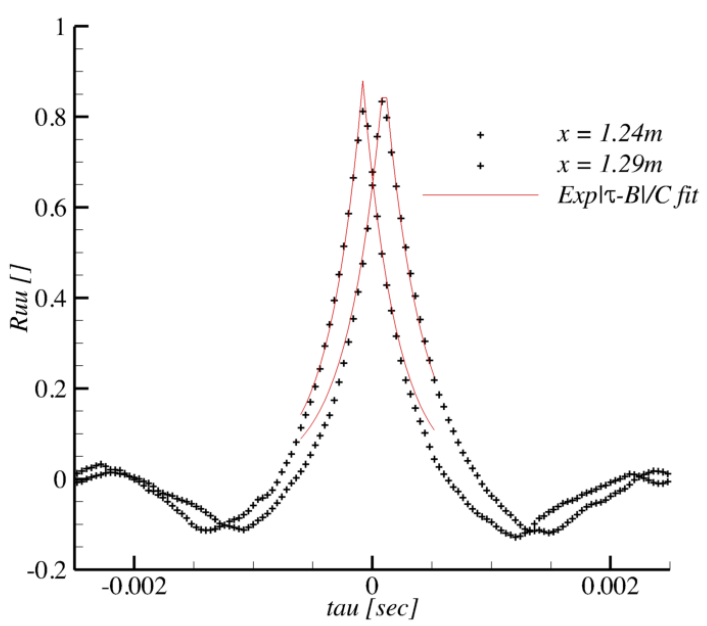

(b)

Figure 6 Example of space-time correlation of axial velocity near centerline of jet from TRPIV data. Line plot on right shows extracted correlation at top and bottom of left plot with fitted curves to find peaks. Values for $B(x)$ of the two fitted curves, $-0.00004(1.240), 0.000110(1.290)$, produce a convection speed of $333 \mathrm{~m} / \mathrm{s}$.

\section{B. Profiles of Convection Speed}

In the following section, groups of plots are created for each nozzle and flow combination, one for each axial station, combining the transverse profiles of mean velocity $\left\langle u>/ U_{\mathrm{j}}\right.$, mean square turbulent velocity $\left\langle u u>/ U_{\mathrm{j}}^{2}\right.$, and convection speed $U_{c} / U_{j}$ on each plot. For each point in the profile, a space-time correlation was performed for that radial location, and the slope of the peak found using a fitting routine. There are some regions where the convection speed results change rapidly in space, typically where there is little turbulent energy (such as in the potential core), and there the result must be considered void. Because data was acquired with two or three overlapping fields of view there are multiple data in the regions of overlap, providing some guide to confidence in the measurements.

There are two trends to observe in all the plots: (1) in portions of the flow where there is significant turbulence (where the convection speed actually has meaning), the convection speed closely follows the mean velocity, (2) in flows with an axisymmetric nozzle the mean axial velocity profile is symmetric; however, the turbulent velocity profile is often not.

The first observation is important because previous convection measurements, taken in the potential core region of the jet, indicate that convection velocities do not vary as much as the mean velocity across the jet. Applied to the entire jet, this is incorrect. The measurements made in the potential core of the cold single-stream jet (Figure 7) exemplify this. At axial locations in the potential core $\left(x / D_{j}<7\right)$ the convection velocity profile matches the mean velocity profile at the radial location of peak turbulence and is lower (at lower radii) or higher (at higher radii) than the mean velocity elsewhere. Downstream of the potential core, however, the convection velocity and mean velocity are very close.

The key to the relationship between the convection velocity and the mean velocity is the amplitude of the turbulence. Looking over all the profiles it is clear that in regions where there is little turbulent energy the convection speed that is measured is actually that of the nearby turbulent region. The classic value of $U_{\mathrm{c}}=0.62 U_{0}$ is 
a reflection of the fact that the turbulent energy peaks around the radial location where $<u>=0.62 U_{0}$. A more general finding would be $U_{c}=\langle u\rangle$ and in most applications it won't matter that this is not correct in regions of low turbulence, as these regions won't contribute to the turbulence-generated phenomena anyway. Perhaps a more general model for the convection velocity $U_{c}(y)$ would include an average of the local mean velocities weighted by their turbulence levels and distance from the point of interest.

The second observation adds to the growing evidence ${ }^{19,20}$ that $T K E$ is more sensitive to asymmetries in the geometry than mean velocity would indicate. This may explain why turbulence-generated quantities, such as noise, can be sensitive to slight variations in the build of the model. Within the measurements shown here the convection speed is not as sensitive to asymmetries as the turbulence.

\section{Single-stream jets}

These general trends mentioned, consider the data. Figure 7 - Figure 9 show the measurements made in singlestream unheated and heated jets. First, the wake of the internal plug is evident in the mean velocity profiles near the exit, a feature that is not found in simple nozzles without a centerbody. Second, compared with classic hotwire measurements of convection velocity profiles (Figure 1) the profile for $U_{\mathrm{c}}$ matches that of the hotwire measurements over the region where significant turbulence exists. The convection speed $U_{\mathrm{c}}$ matches the mean velocity $\langle u\rangle$ at the location of peak turbulence around $U_{\mathrm{c}}=0.6 U_{0}$, being slightly higher than the local mean velocity on the low speed side and slightly lower on the high-speed side, as noted above. Third, there is an interesting local increase in 'convection speed' well outside the jet near the nozzle $\left(x / D_{\mathrm{j}}<6\right)$, although what is being convected is not clear. Fourth, in the potential core region $\left(x / D_{\mathrm{j}}<6\right)$ a convection speed is registered near the centerline which may be the turbulence of the plug wake. Fifth, and most importantly, there is no significant difference in the relationship between local mean velocity and convection speed in the heated and unheated single-jet cases. All of these observations hold for hot and cold jets. 

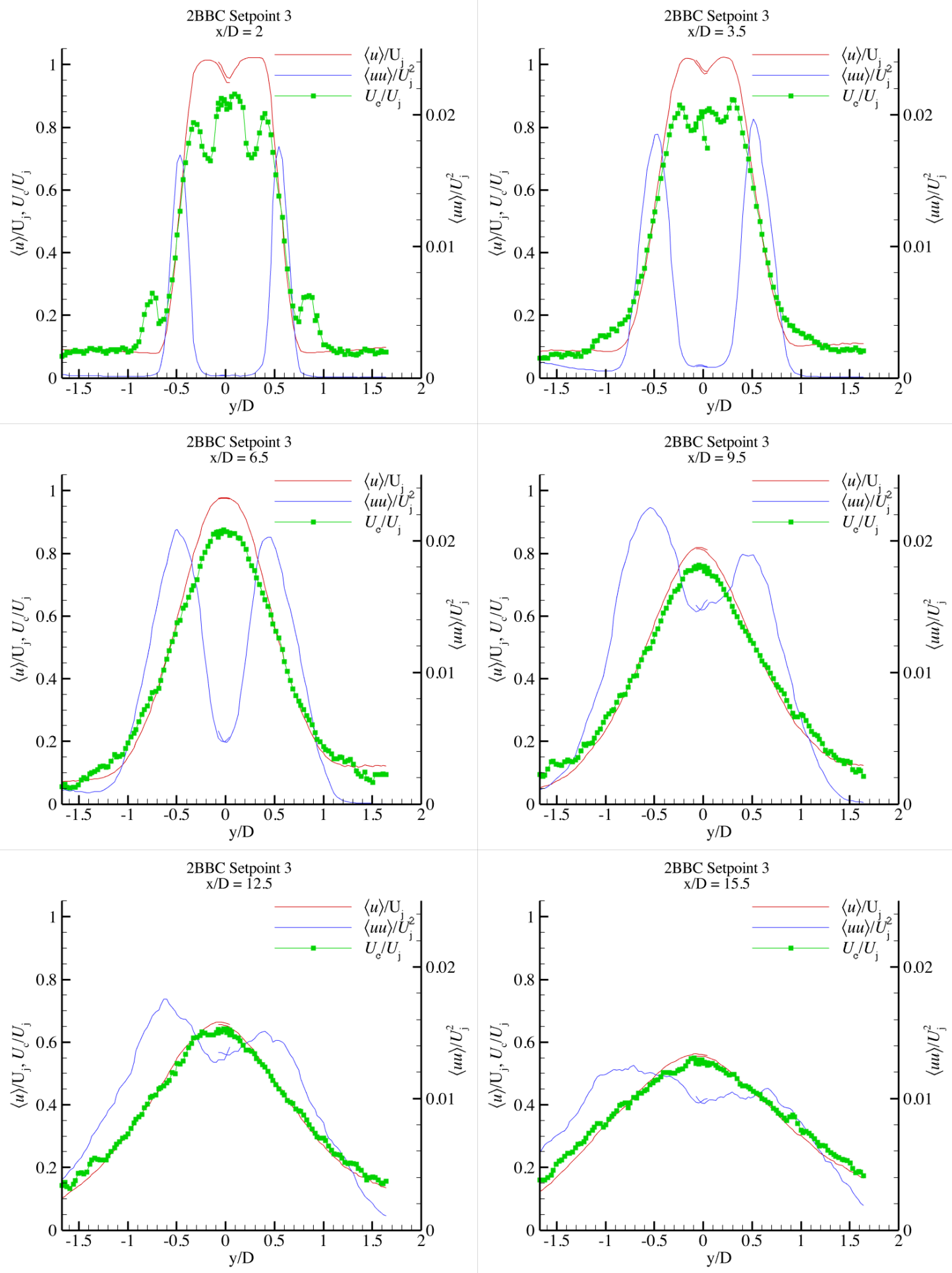

Figure 7 Transverse profiles of mean velocity, variance of turbulent velocity, and convection speed for six axial stations. Jet is single-stream plume from nozzle 2BBc at setpoint 3 (unheated Ma $=0.5$ ). 

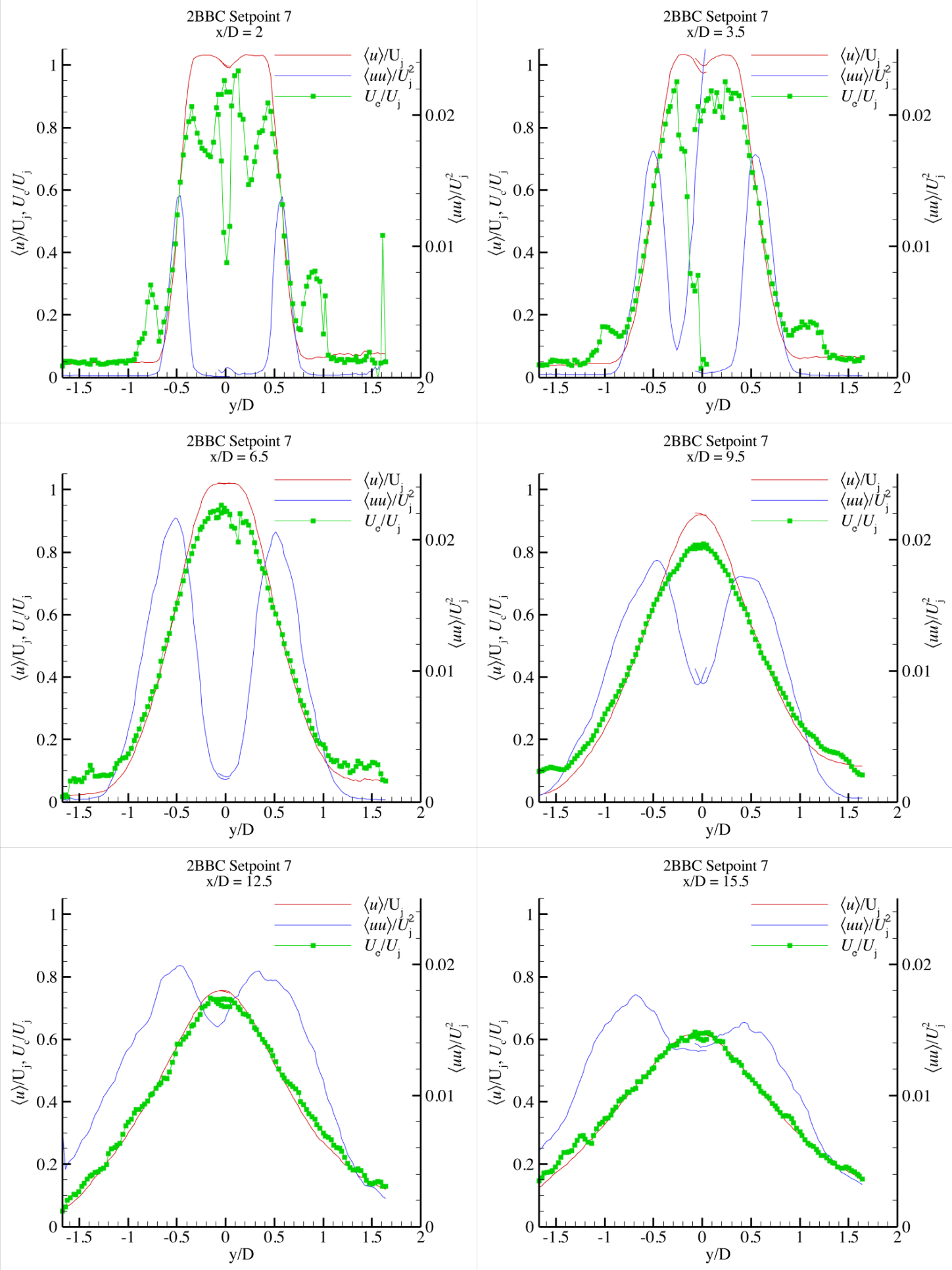

Figure 8 Transverse profiles of mean velocity, variance of turbulent velocity, and convection speed for six axial stations. Jet is single-stream plume from nozzle 2BBc at setpoint 7 (unheated Ma $=0.9$ ). 

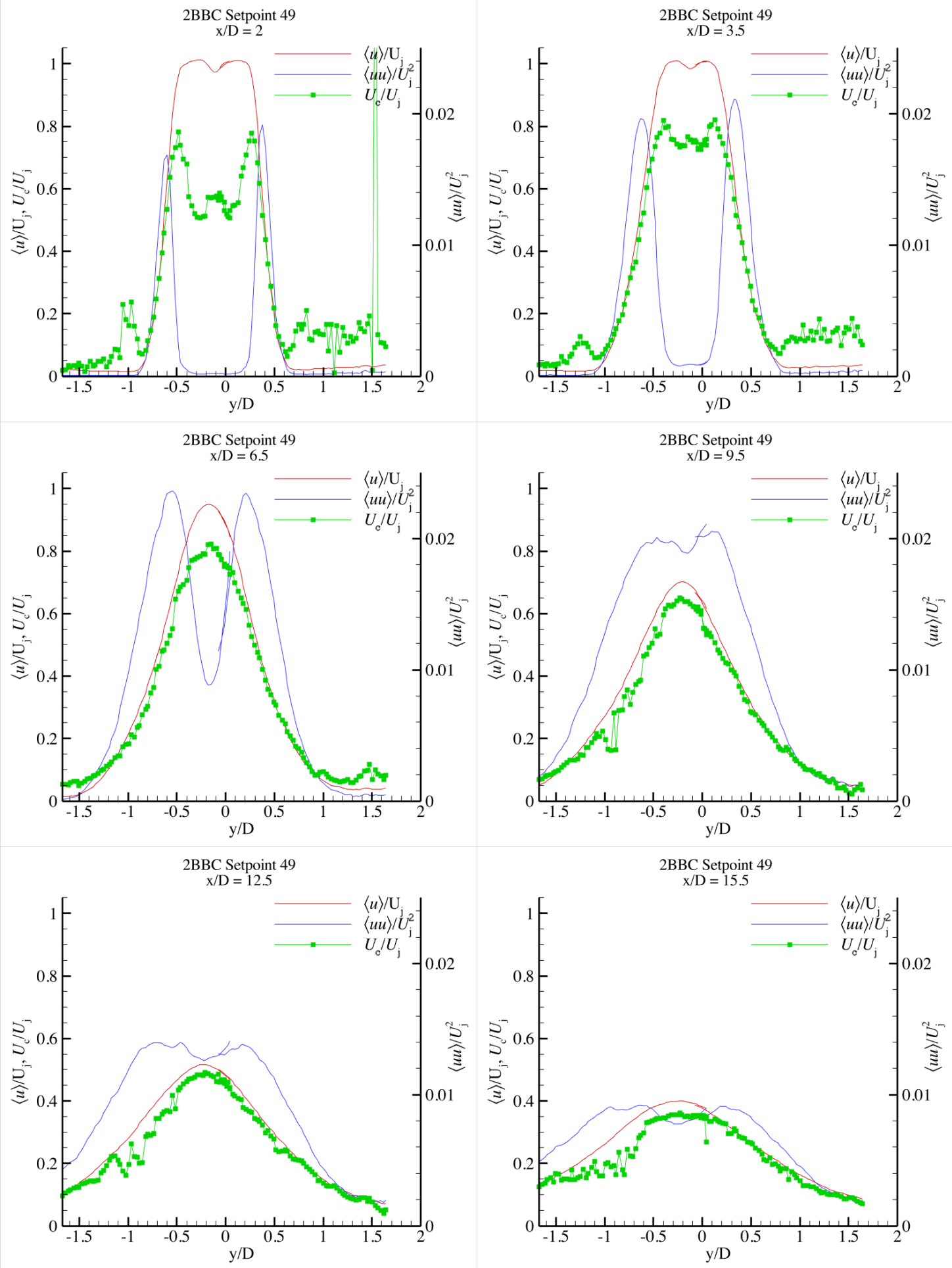

Figure 9 Transverse profiles of mean velocity, variance of turbulent velocity, and convection speed for six axial stations. Jet is hot single-stream plume from nozzle $2 B B c$ at setpoint $49\left(M a=1.48, T s / T_{\infty}=2.7\right)$.

\section{Multistream axisymmetric jets}

Turning to the cases of multi-stream jet plumes, the picture becomes messier. With three concentric streams the mean profiles show multiple inflection points, and often the profiles are not symmetric, even in the cases of 'axisymmetric' nozzles (Figure 11 - Figure 17). These figures feature variations in effective area ratio (Figure 12 vs Figure 12), and various initial velocity stream profiles. Figure 13 features initial jet conditions with all unheated 
streams and monotonically changing initial velocities down to a static ambient speed. Figure 14 through Figure 16 show variations in stream conditions on all three streams relative to Figure 10.

First, some common features found in all these axisymmetric, multistream cases. First, note that the plug wake is much pronounced here because the plug is external and the measurements are made much closer to the plug. The convection speeds measured near the centerline near the nozzle are presumably more reflective of the plug wake and are significantly lower than the local mean velocity. Given how low $\langle u u\rangle / U_{\mathrm{j}}^{2}$ is in these locations, it is possible that there are multiple turbulent sources nearby (plug wake, shear layer) imposing their unsteadiness on the essentially laminar flow in these regions. In regions where there is significant turbulence, the convection speed measurements are smooth and more nearly match the mean velocity profile.

Second, the convection velocity in the flight stream is measured to be much lower than the local mean. This is a puzzle because there is relatively little turbulence in the flight stream to have its own convection velocity compared to the induced perturbations from the higher speed shear layer nearby. Perhaps the answer to this puzzle will also address the increases in local convection speed just outside the static jet plumes, noted in the single-stream jets and found again in the static multi-stream jets (Figure 12 and Figure 13). Both are likely due to hydrodynamic fluctuations imposed on the nearly laminar flow in these regions. At this time no answer presents itself.

Even though three coaxial streams of air were flowing in most of the cases shown in this section, only one shear layer is detected at the first axial measurement point. As can be seen in Figure 3, the third stream of the nozzle emits from a very narrow annulus far upstream; its shear layer relative to ambient is not seen in the data.

It has been noted ${ }^{20}$ before that in multi-stream flows where the mean velocities are apparently initially axisymmetric, the turbulence levels are often not. In the flows here it appears that there is an asymmetry in the plug wake or the innermost nozzle separating the first two inner streams. In all the cases shown in Figure 11-17 the profile of $\left\langle u u>/ U_{\mathrm{j}}^{2}\right.$ is symmetric near the nozzle $(\mathrm{x}<0.6 \mathrm{~m})$, where the shear layer between the outer flow of the jet and the ambient dominate. The profiles are not symmetric in the innermost regions, where the shear is generated by the two innermost flows. The profiles of $\langle u u\rangle / U_{\mathrm{j}}^{2}$ become asymmetric farther downstream when the innermost shear layer merges with the outermost. This is easily seen in Fig 12, panels (c)-(f).

Although not documented in this test, the nozzle and rig hardware have generally been found to have very small deviations (less than $0.1 \mathrm{~mm}$ ) from concentricity. Unfortunately, schedule restrictions did not allow a followup test, with a carefully documented rig buildup, to explore this further. One other potential source of asymmetry was the ambient flow. Measurements were taken in the vertical plane of the rig, which contains the air feed pipes covered by an airfoil shell. The wake of this strut might have influenced the negative-y side of the jet. However, comparing (with flight stream) and Figure 12 (without flight stream), it seems that a wake in the flight stream is not likely to be the source of the asymmetry: the asymmetries are very similar with or without the flight stream. 

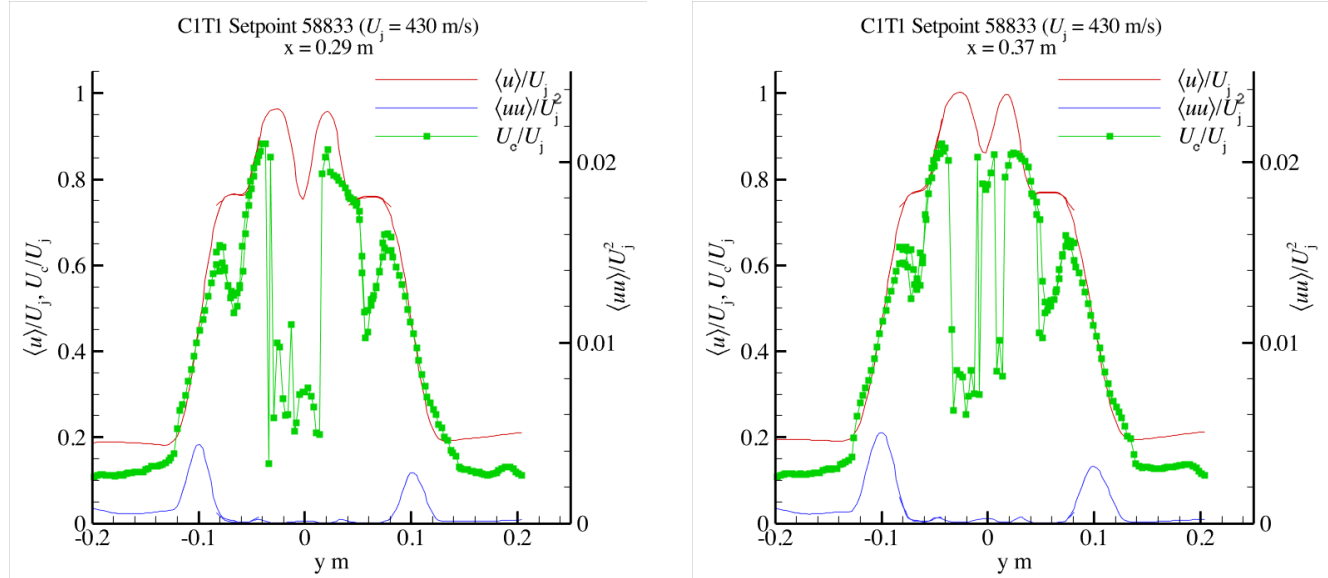

(a)

(b)
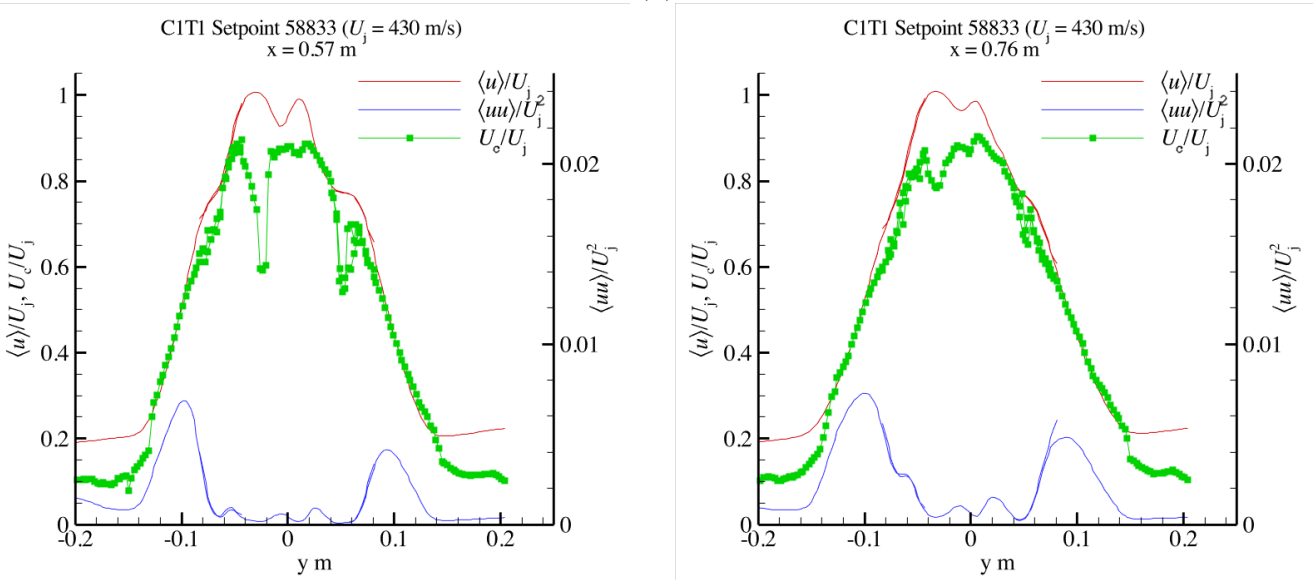

(c)

(d)
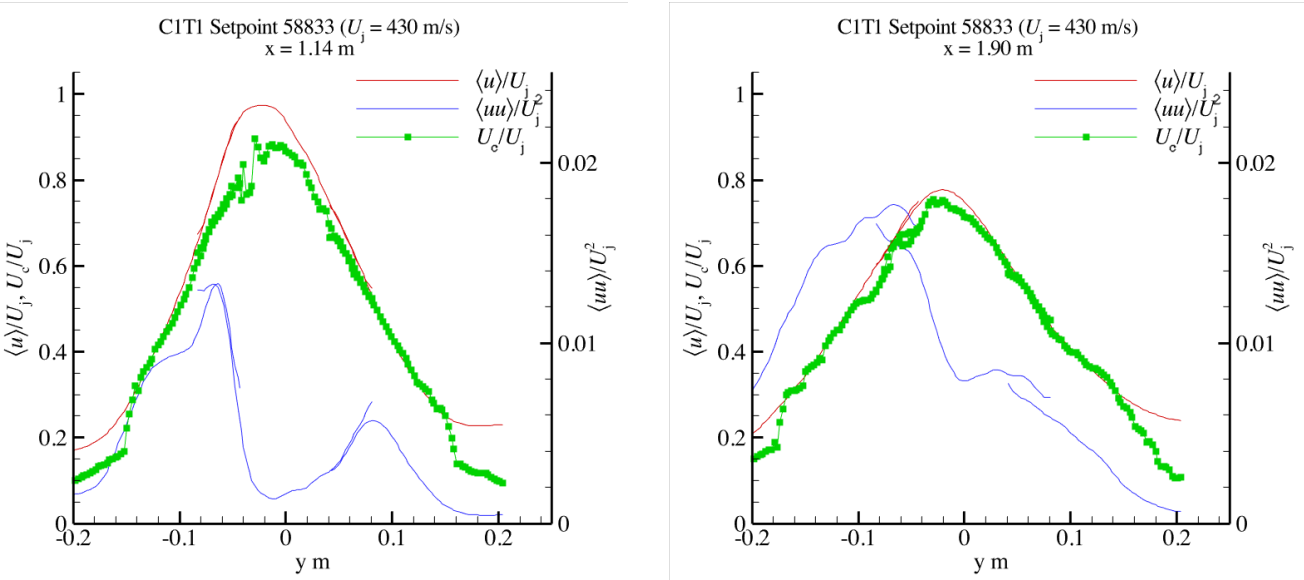

(e)

(f)

Figure 10 Transverse profiles of mean velocity, variance of turbulent velocity, and convection speed for eight axial stations. Jet is dual-stream plume from nozzle C1T1AXI at setpoint 58833. 

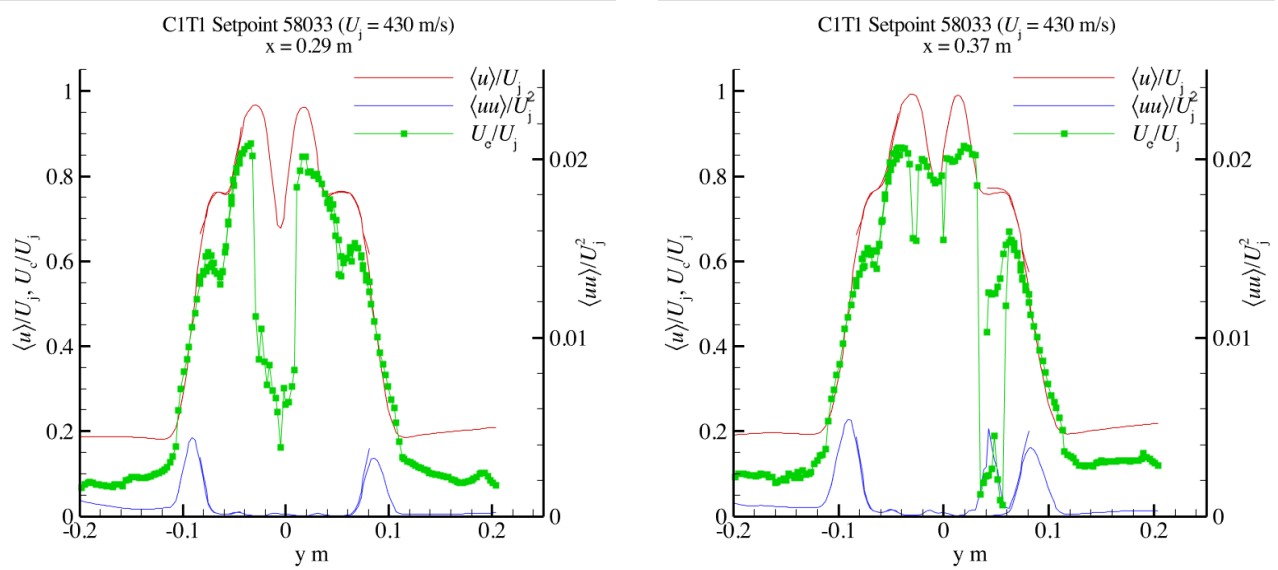

(a)

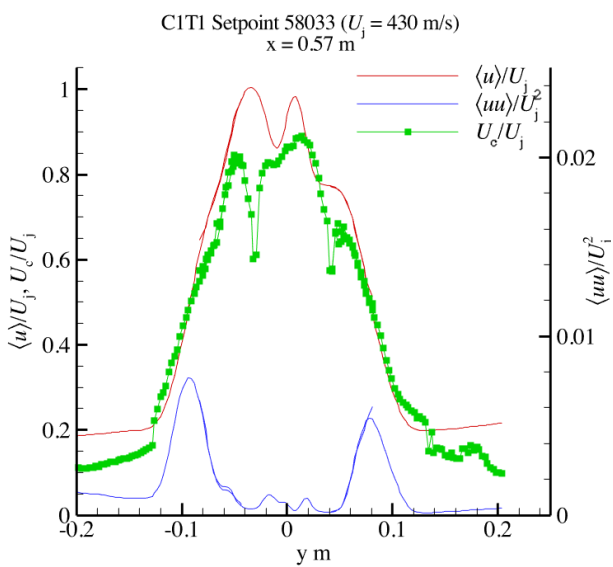

(c)

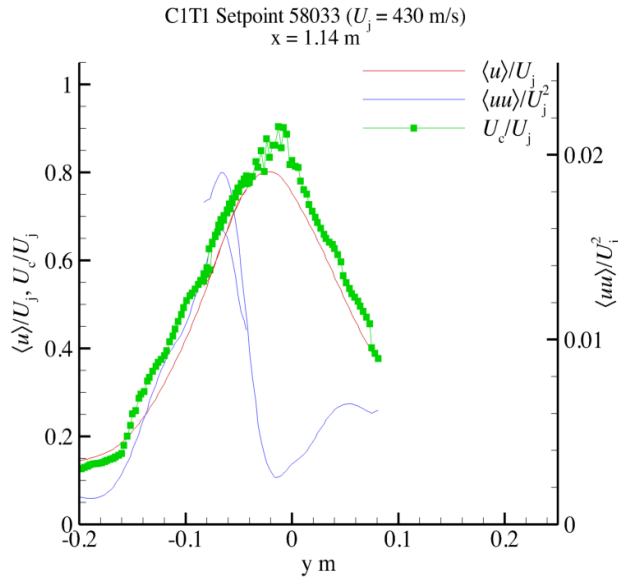

(b)

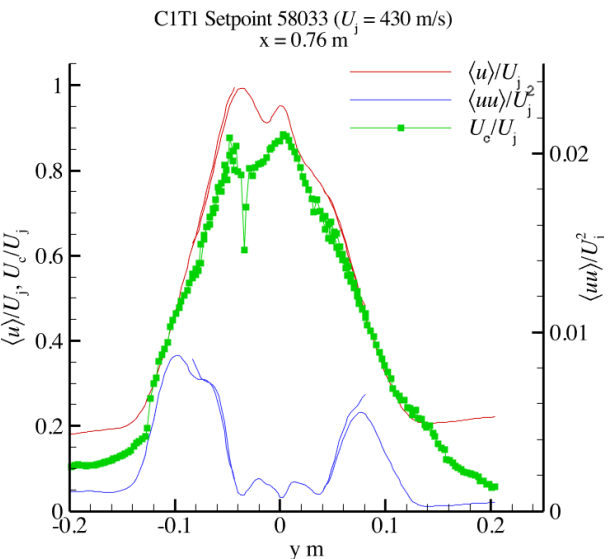

(d)
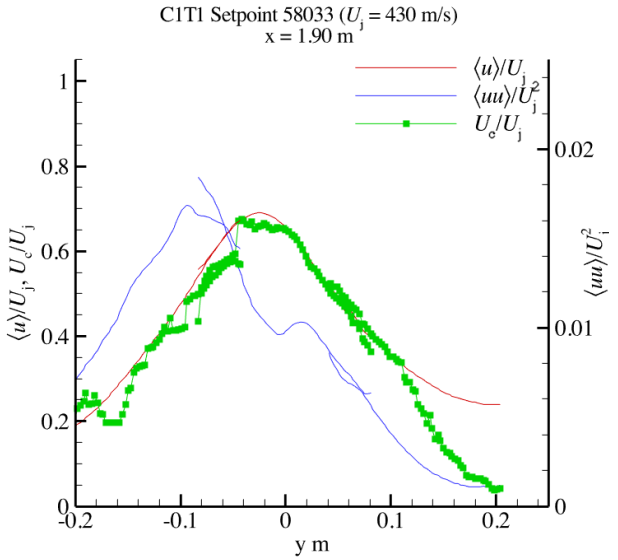

(f)

Figure 11 Transverse profiles of mean velocity, variance of turbulent velocity, and convection speed for eight axial stations. Jet is dual-stream plume from nozzle C1T1AXI at setpoint 58033. 

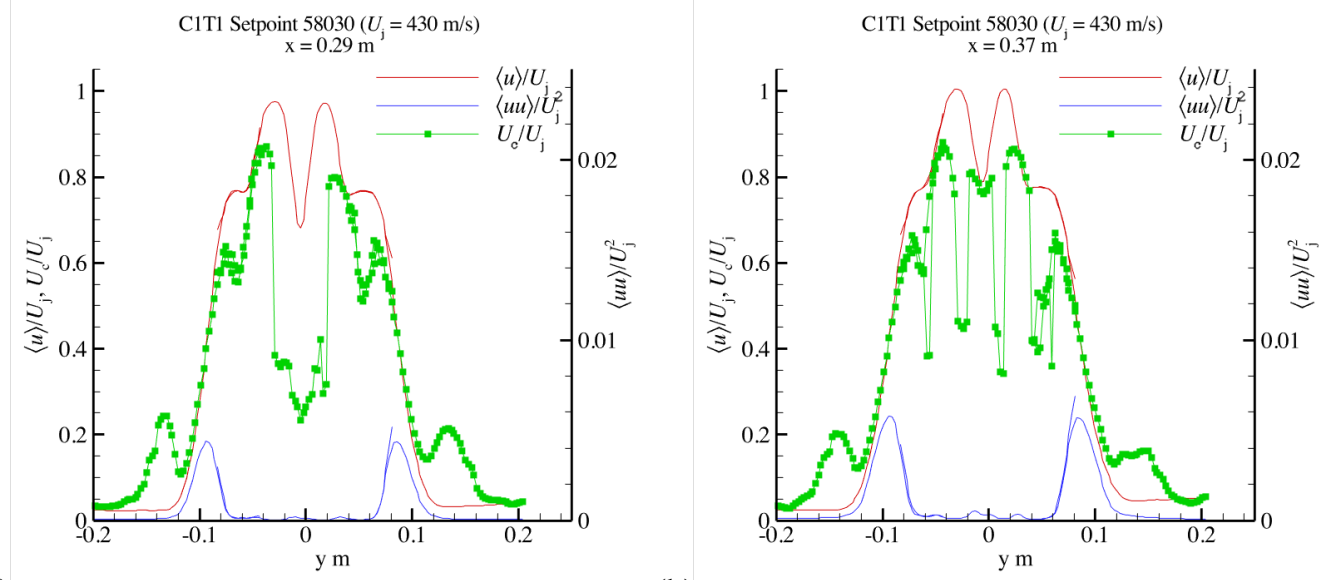

(a)

(b)
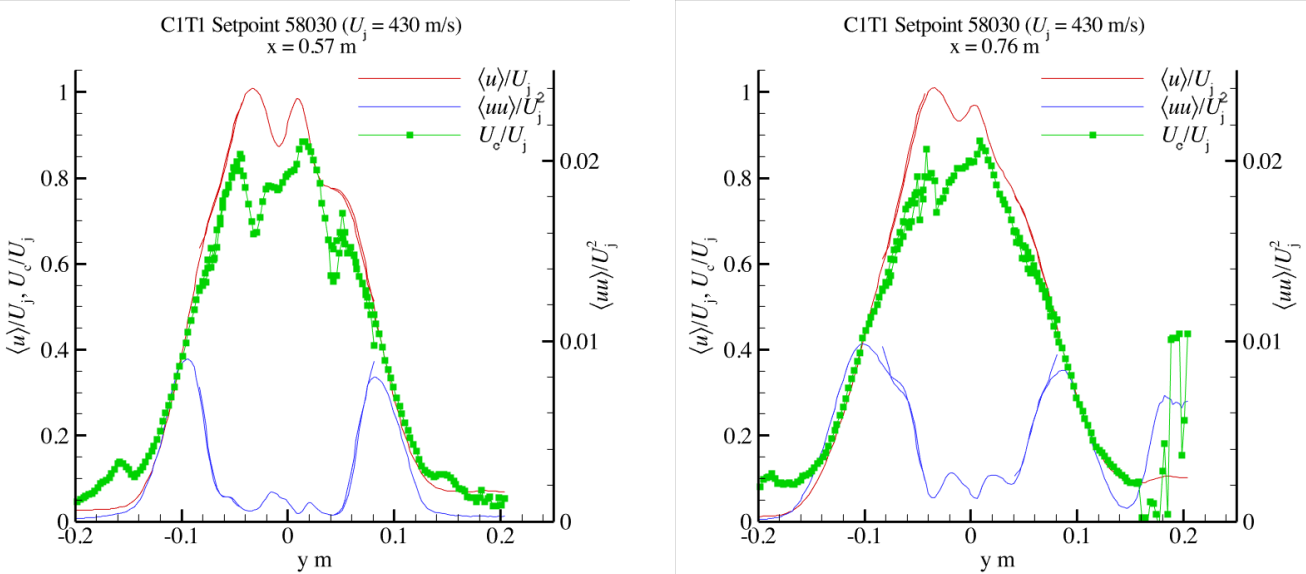

(c)

(d)
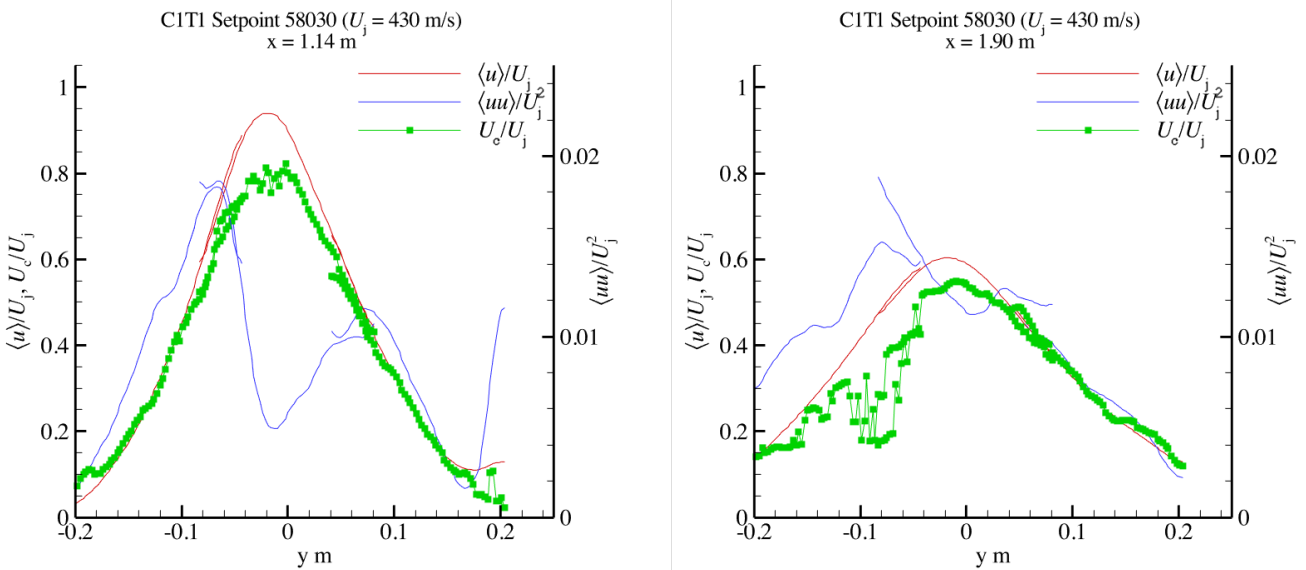

(e)

(f)

Figure 12 Transverse profiles of mean velocity, variance of turbulent velocity, and convection speed for eight axial stations. Jet is dual-stream plume from nozzle C1T1AXI at setpoint 58030. 

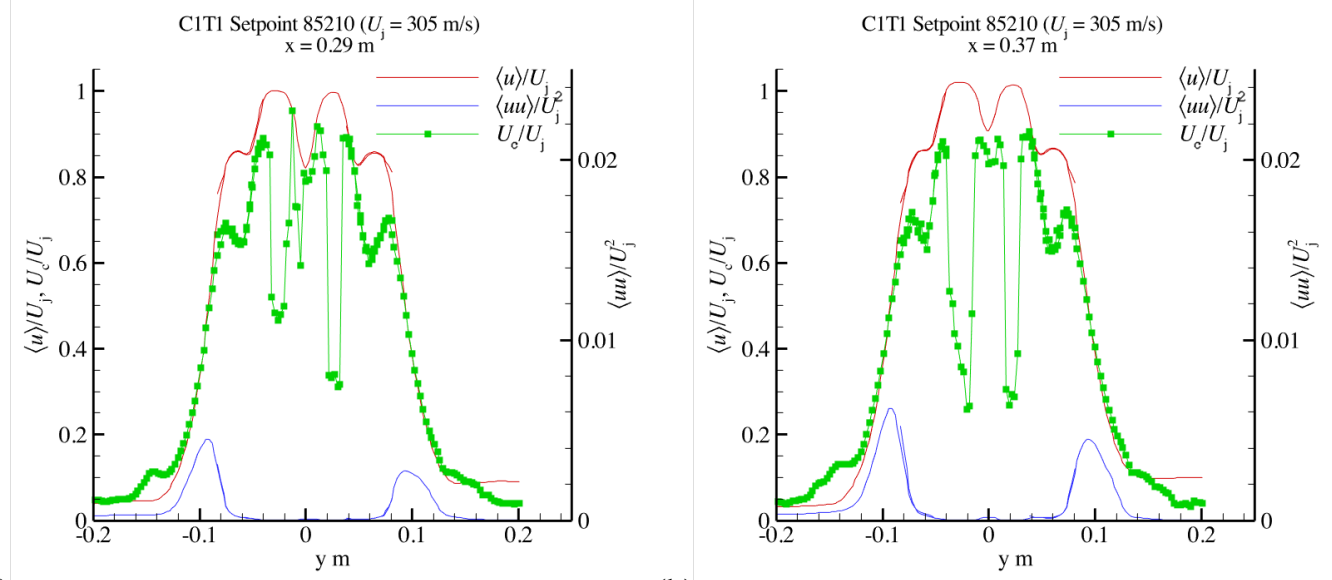

(a)

(b)
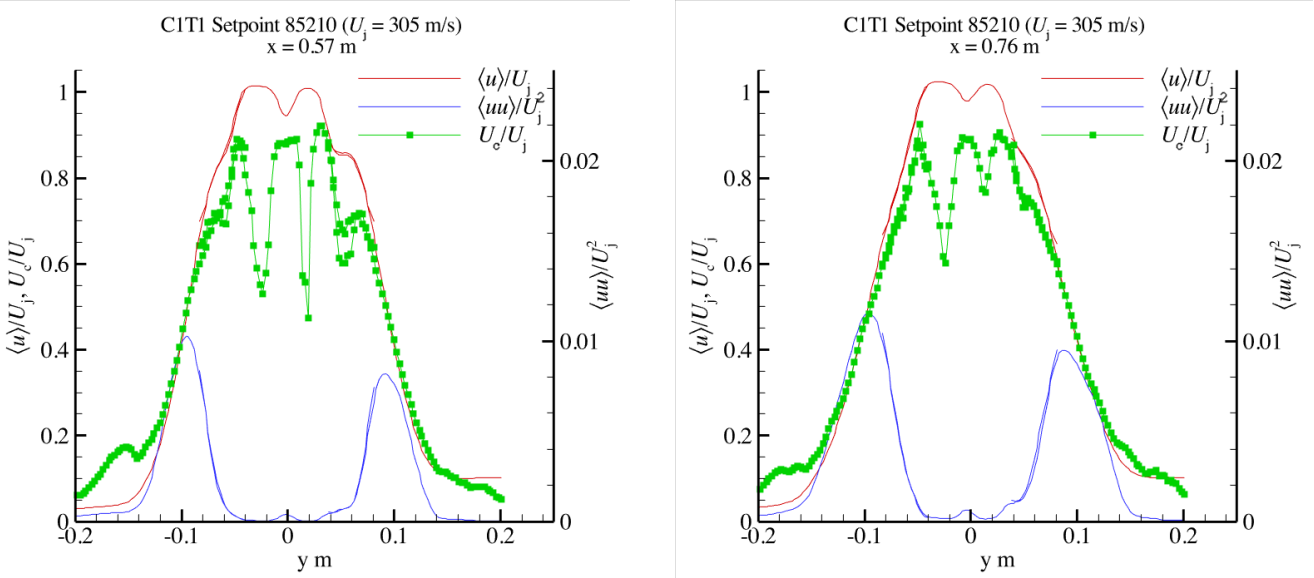

(c)

(d)
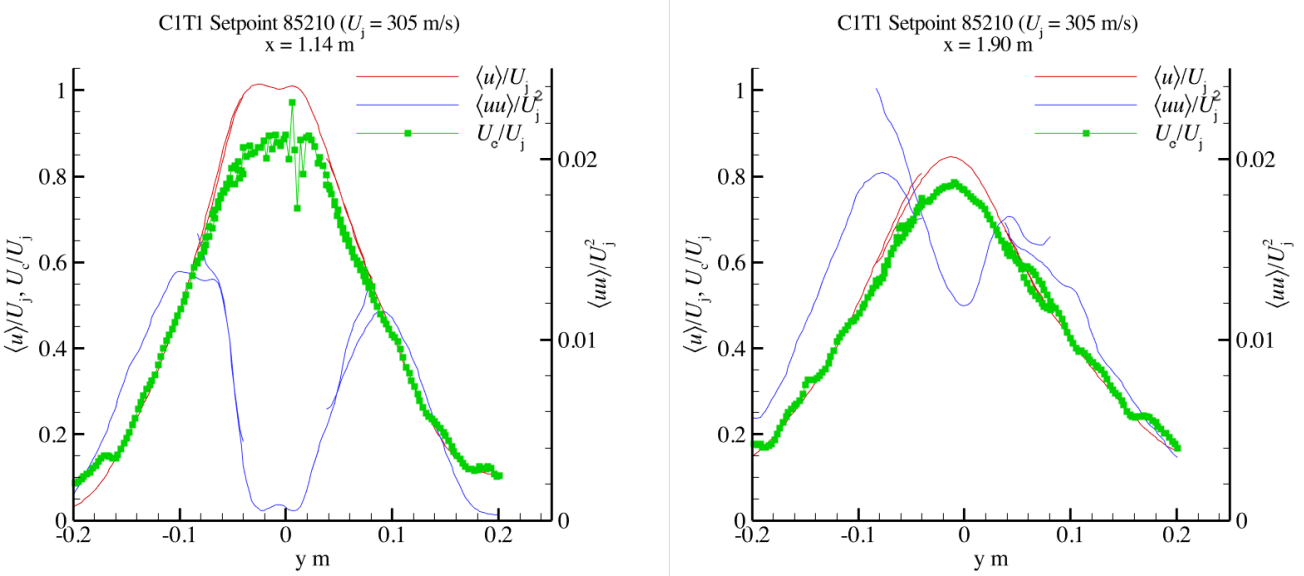

(e)

(f)

Figure 13 Transverse profiles of mean velocity, variance of turbulent velocity, and convection speed for eight axial stations. Jet is dual-stream plume from nozzle C1T1AXI at setpoint 85210. 

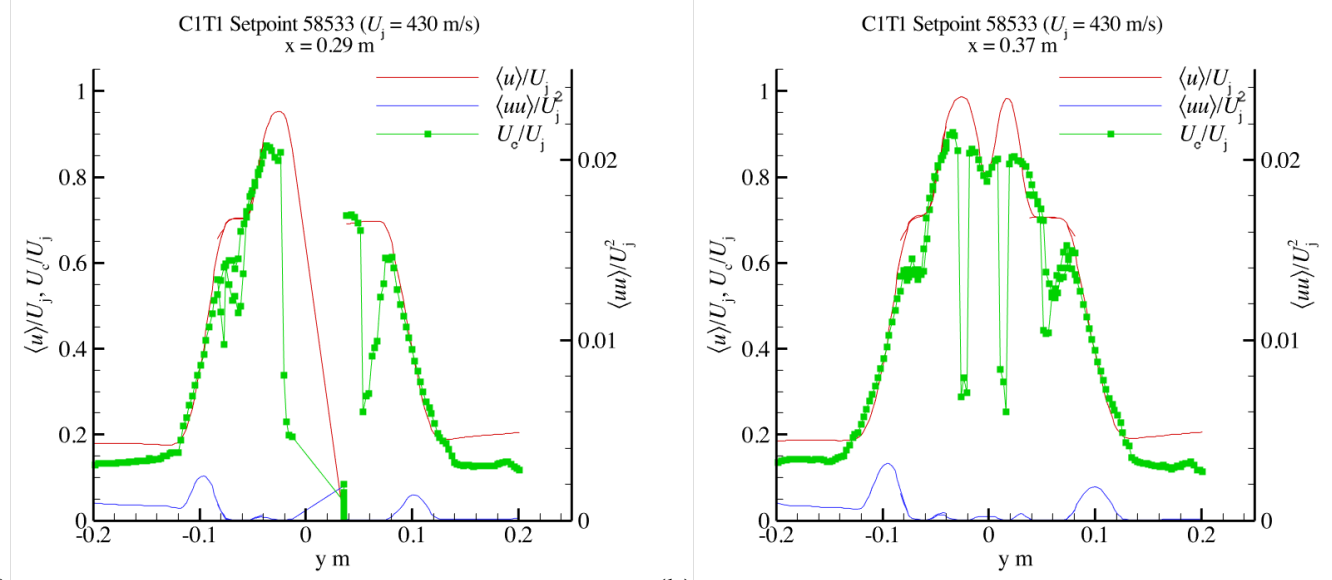

(a)

(b)
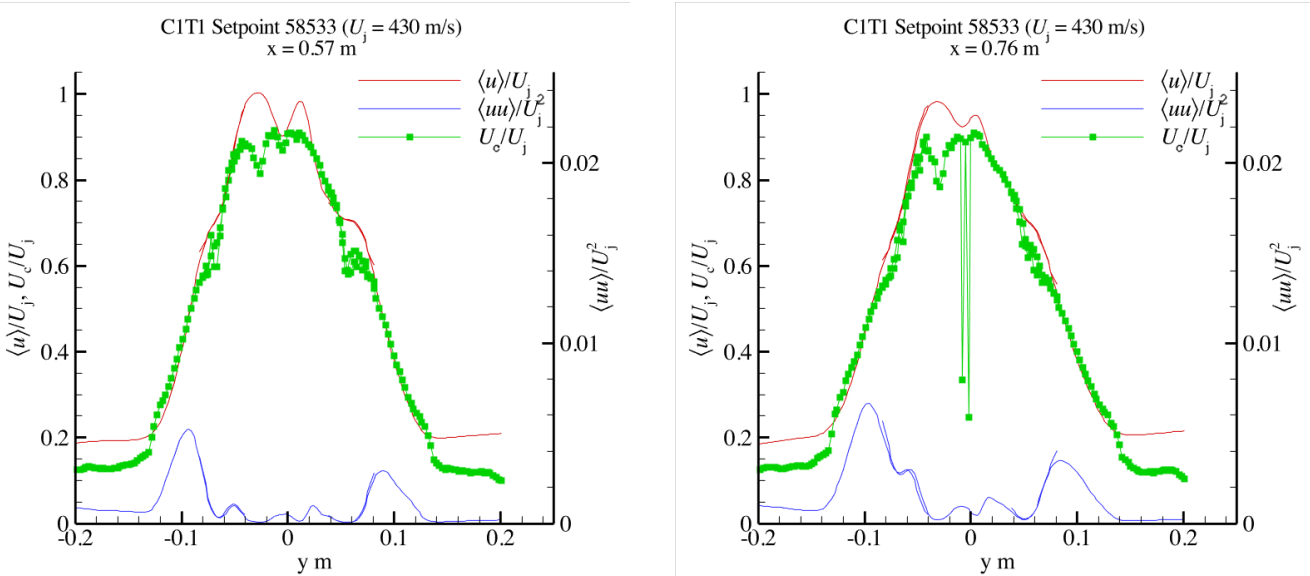

(c)

(d)
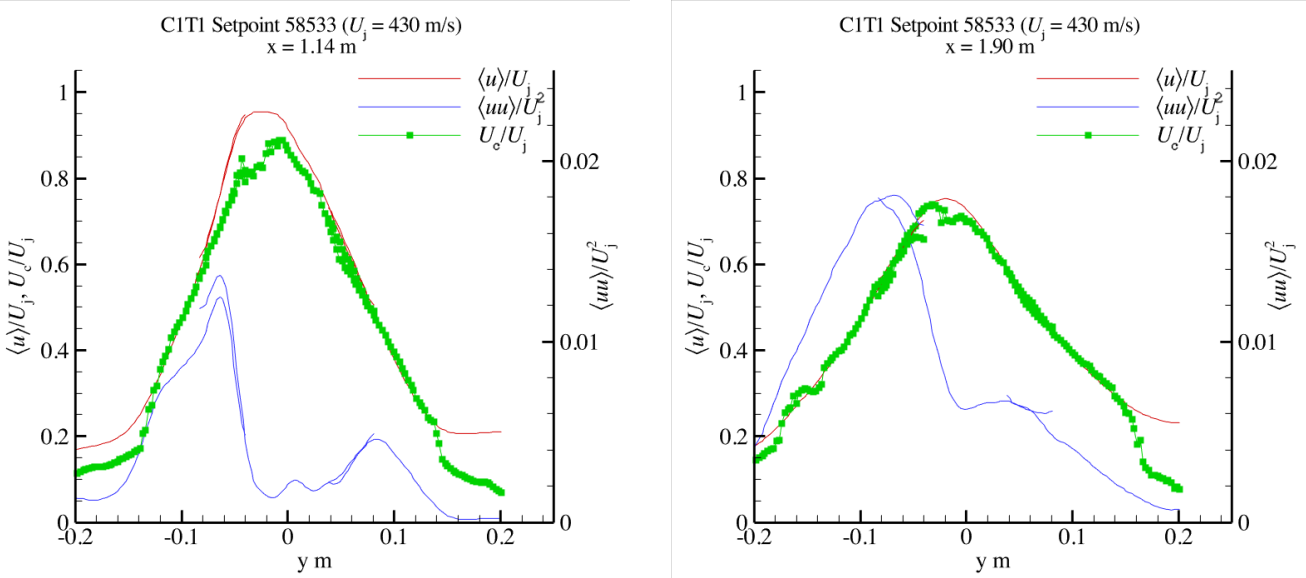

(e)

(f)

Figure 14 Transverse profiles of mean velocity, variance of turbulent velocity, and convection speed for eight axial stations. Jet is dual-stream plume from nozzle C1T1AXI at setpoint 58533. 

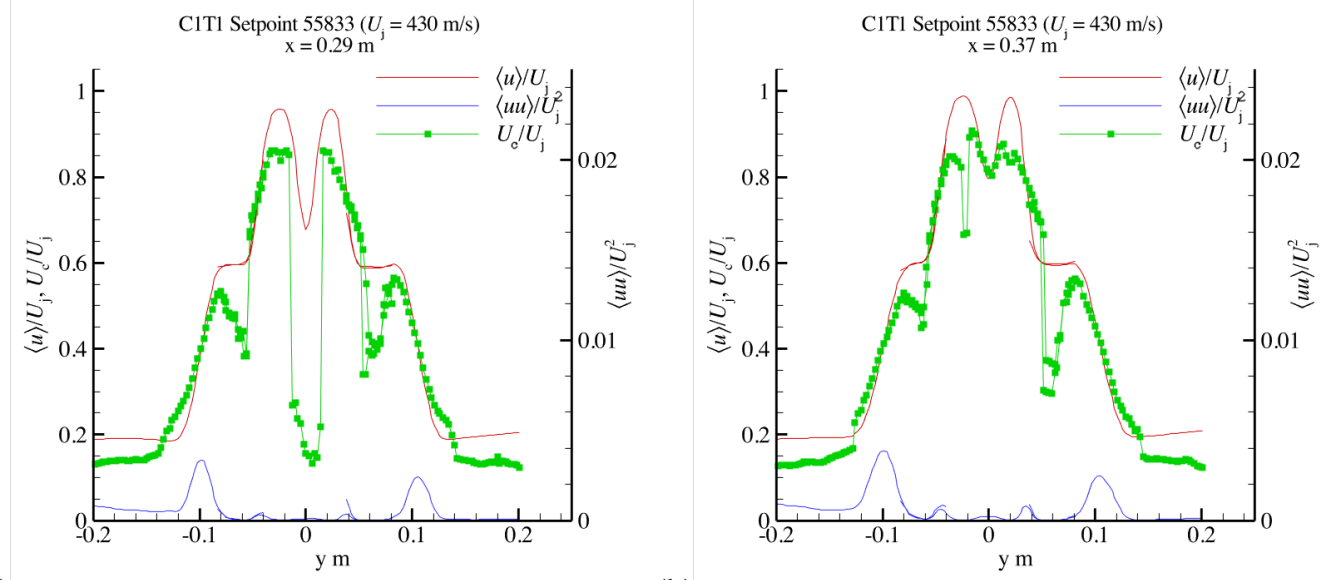

(a)

(b)
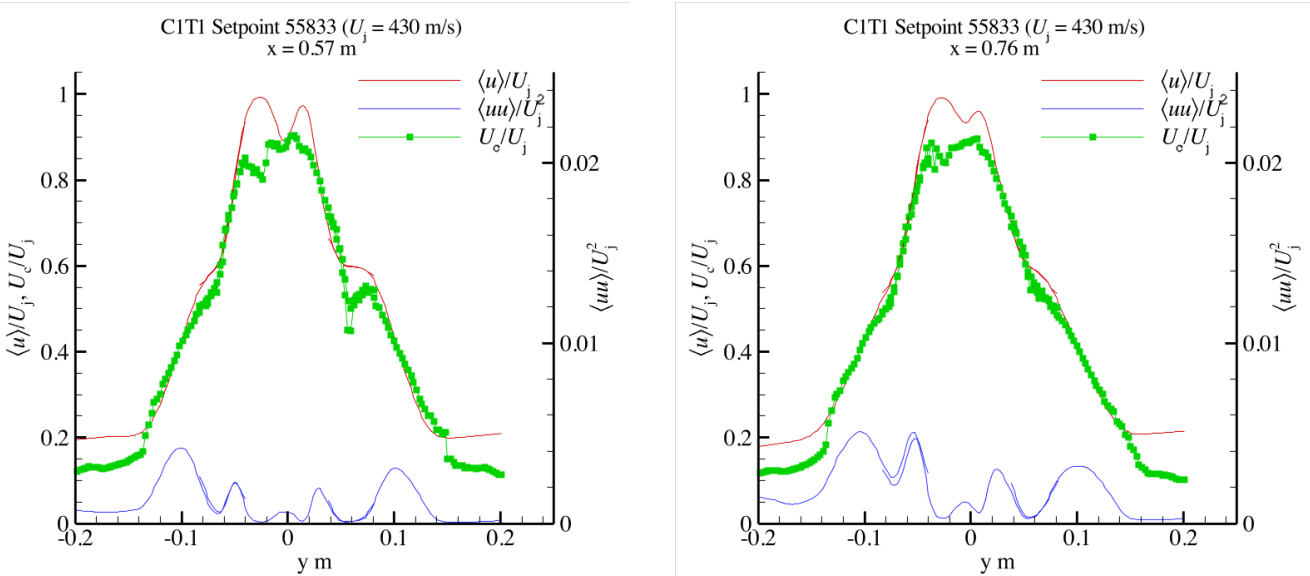

(c)

(d)
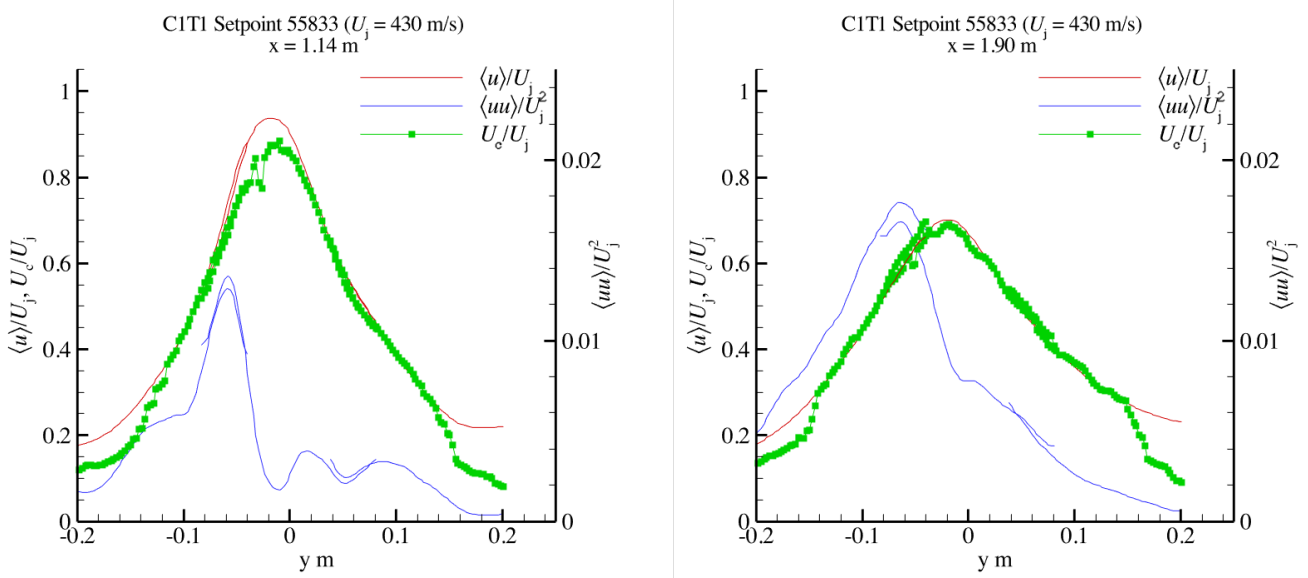

(e)

(f)

Figure 15 Transverse profiles of mean velocity, variance of turbulent velocity, and convection speed for eight axial stations. Jet is dual-stream plume from nozzle C1T1AXI at setpoint 55833. 


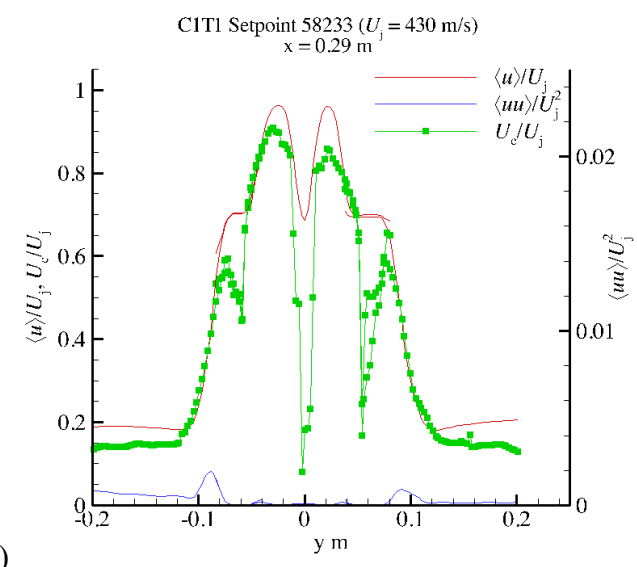

(a)

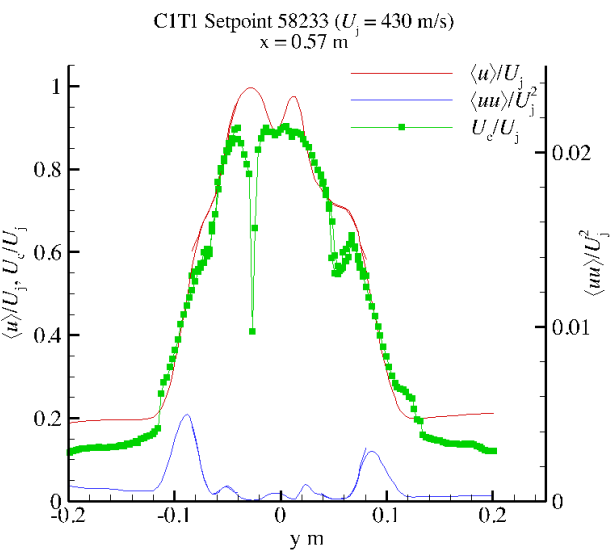

(c)

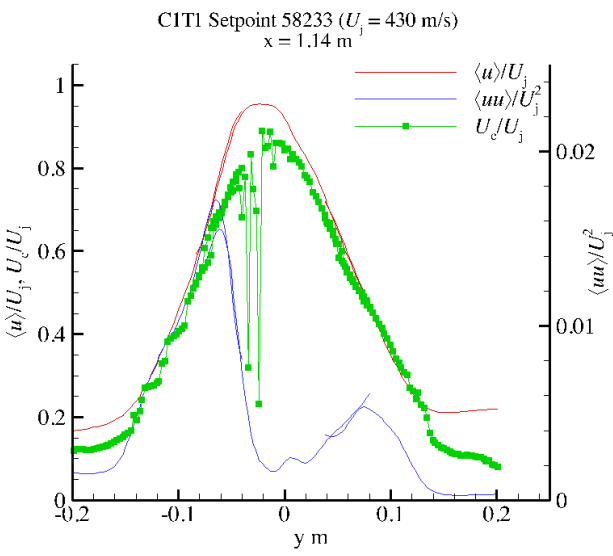

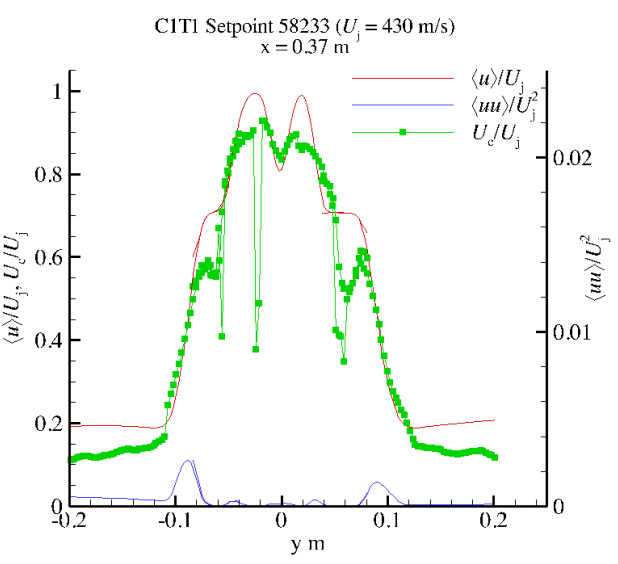

(b)

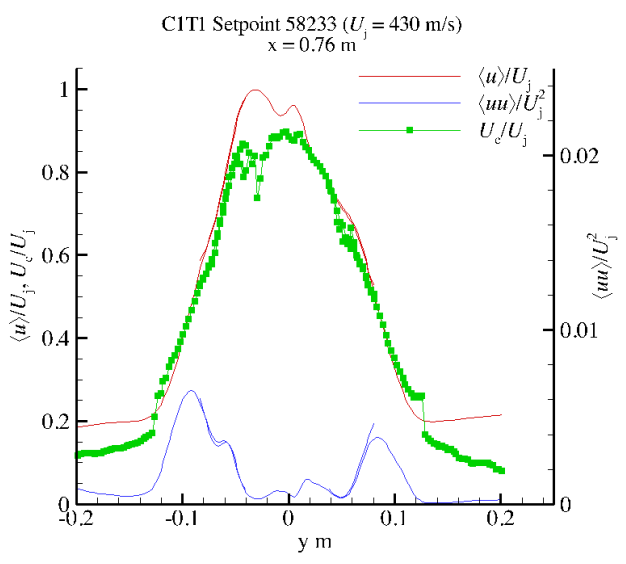

(d)

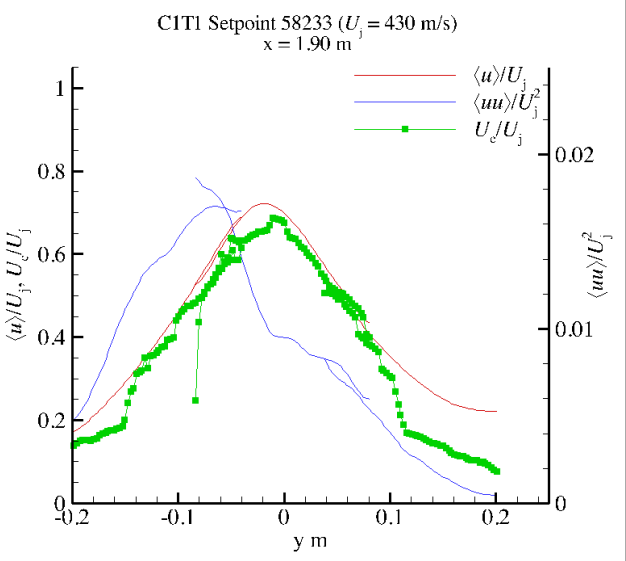

Figure 16 Transverse profiles of mean velocity, variance of turbulent velocity, and convection speed for eight axial stations. Jet is dual-stream plume from nozzle C1T1AXI at setpoint 58233.

\section{Multistream, non-axisymmetric jets}

Turning to the nozzles with a deliberate asymmetry, created by a non-axisymmetric outer annulus for a third stream (Figure 17 - Figure 22), the results are a little bit of a letdown. The profiles are not that much different than the axisymmetric nozzle flows. And the relationships between local mean velocity and convection speed still hold. As noted before, the third stream nozzle annulus was relatively thin and far enough upstream that it did not have a strong impact on the plume. Having the outer stream be significantly asymmetric did have a small effect, but noticeable mostly in contrast with the same flows in the axisymmetric nozzle.

Figure 17 through Figure 19 were taken in the S-duct (SDCT) nozzle where the third stream annulus varies continuously around the periphery of the nozzle. Figure 20 through Figure 22 were taken in the partially blocked 
third stream nozzle (PART) where the 'thin' side actually has no flow from the third stream. In the figures the thicker annulus was on the negative $y$-axis side of the nozzle.

What is consistent is how much the convection speed follows the mean velocity, especially where there is appreciable turbulence. Changing the velocity profile by making the nozzle annuli thicker or thinner did not change the relationship between the local mean velocity, nearby turbulent velocity, and convection speed.

Starting with the S-duct nozzle flows, Figure 17 shows the mean and turbulent velocity profiles and the convection speed profiles for a plume with monotonically decreasing velocities, including a static ambient. The broader skirt on the negative-y side of the mean velocity profile at the initial axial station shows the impact of the thicker tertiary stream. This produces a lower level of turbulence on this side of the jet initially. Downstream this asymmetry in $\langle u u\rangle / U_{\mathrm{j}}^{2}$ is reduced. Comparing this offset nozzle result with that of the axisymmetric nozzle (Figure 13) seems to show the asymmetry of the outer stream counters the asymmetry of the core stream to produce a symmetric result downstream!

In Figure 18 the inflection point of the shear layer separating the second and third nozzle streams can be seen on the thick side at the first measurement station. Little turbulence is generated by this shear layer compared to that of the jet-ambient shear layer. This shear layer, quickly driven by the high speed second stream on both sides produces a fairly symmetric profile of turbulence over most of the jet even though the mean profile shows significant asymmetry which drives the peak mean velocity off centerline. When the third-stream flow is increased to the same as the secondary stream (Figure 19) the turbulence peaks are very similar, even if radially different on the thick and thin sides. The inner shear layer, once it develops significant turbulence $(\mathrm{x}>0.57 \mathrm{~m})$ is merged quickly with the outer shear layer on the thin side, but develops independently for quite a distance downstream on the thick side.

Moving to the partially-blocked third stream nozzle (C1T1PART), Figure 20 revisits the cold, static case presented for the other nozzles (Figure 13 and Figure 17). The results are very similar as to the SDCT offset nozzle, producing a bit faster mixing, with higher turbulence levels by the last station measured. The differences between the two offset nozzles for setpoint 58533 (Figure 18 and Figure 21) are also small, the partially blocked nozzle perhaps mixing a bit faster.

Figure 22 shows profiles for a flow condition not shown previously, but may have some importance to the question of which shear layers have the most impact on the convection speed felt by the hydrodynamic field near to the jet. This near-jet field is thought to be key to the generation of large-scale noise. The setpoint 88533 flow is somewhat different than the others in that the core stream is significantly higher velocity than the other two streams, and it might be reasonable to expect the shear layer between this stream and the next fan stream to dominate the dynamics of the jet plume. However, the outer shear layer, between the combined second and third streams, produces much stronger turbulence than the inner shear layer. The inner shear layer only begins to rival the outer in turbulent strength when it merges with the outer shear layer on the thick side. By this point the shear layer has moved radially outward significantly where the mean velocity, and local convection speed, is lower. It is also worth noting that this setpoint was included to push the limits of the analysis, with the high speed convecting the turbulence across the PIV field of view in a very few sample times. The consistency of the measurements at this flow condition show that the assumptions in the design of the experiment were valid and that the analysis method was robust. 

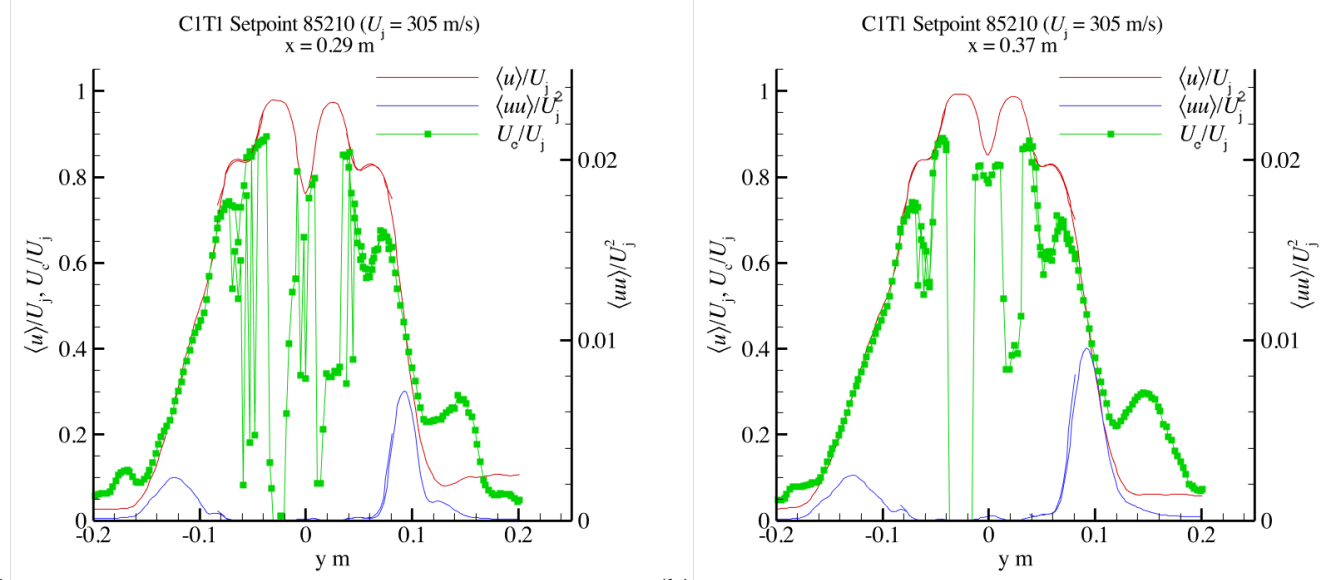

(a)

(b)
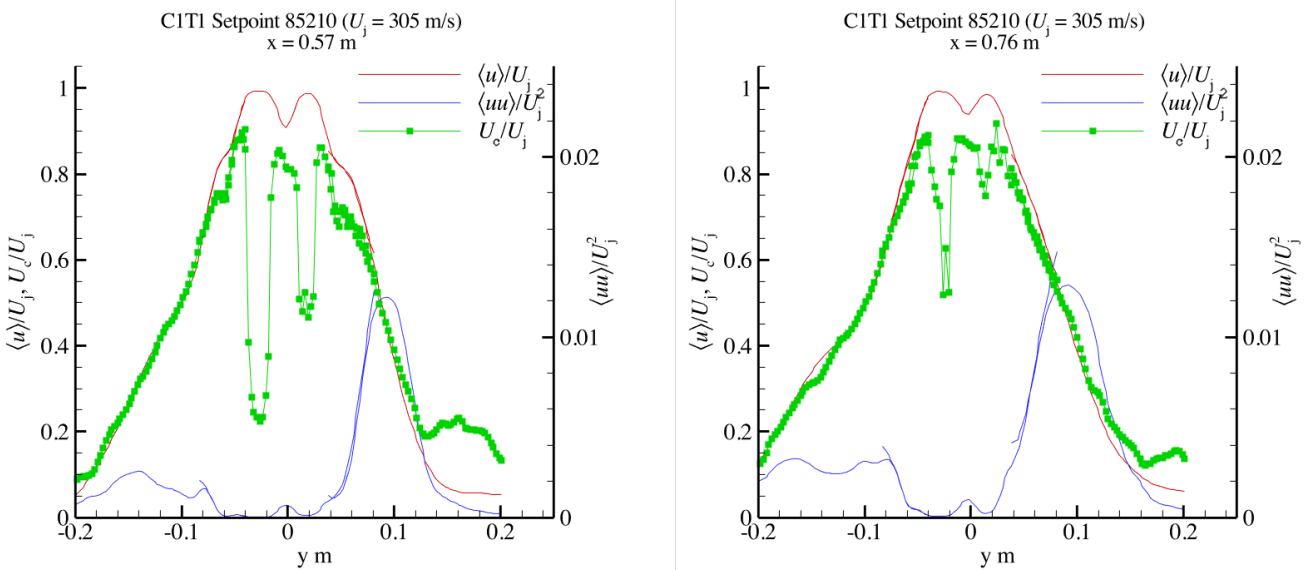

(c)

(d)
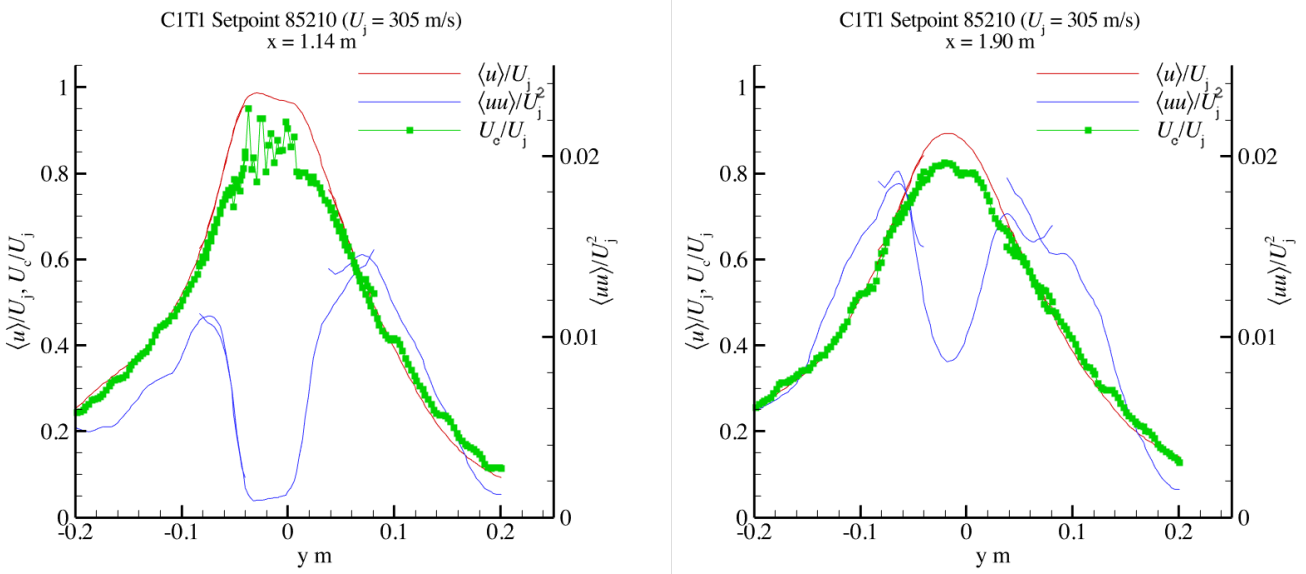

(e)

(f)

Figure 17 Transverse profiles of mean velocity, variance of turbulent velocity, and convection speed for eight axial stations. Jet is dual-stream plume from nozzle C1T1SDCT at setpoint 85210. 

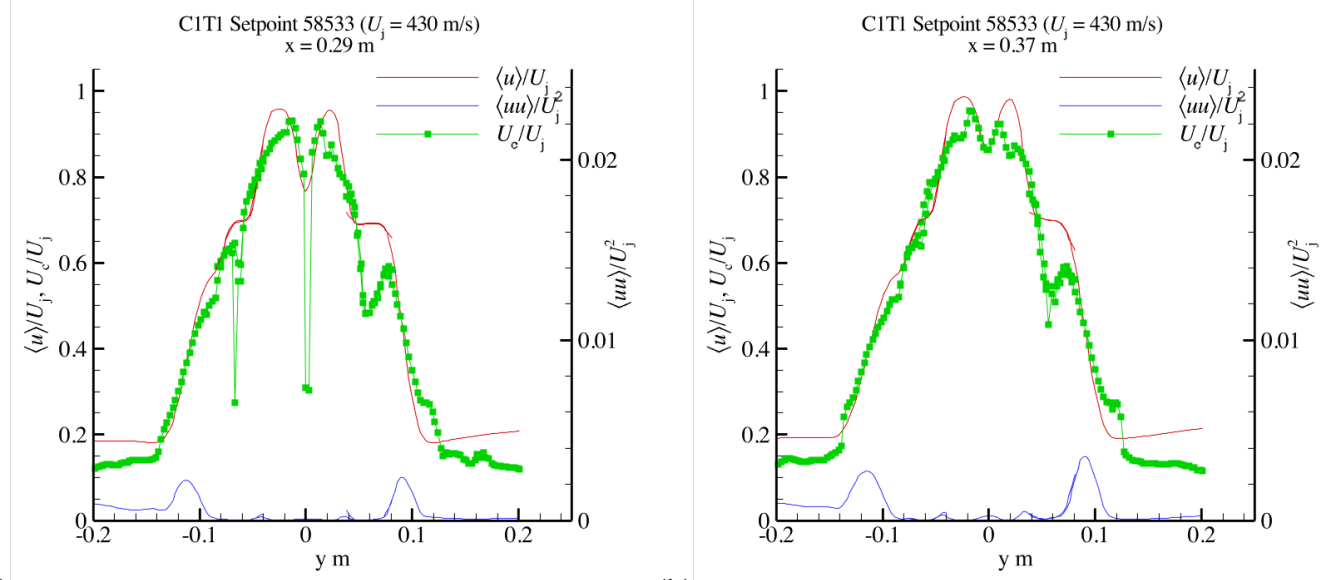

(a)

(b)
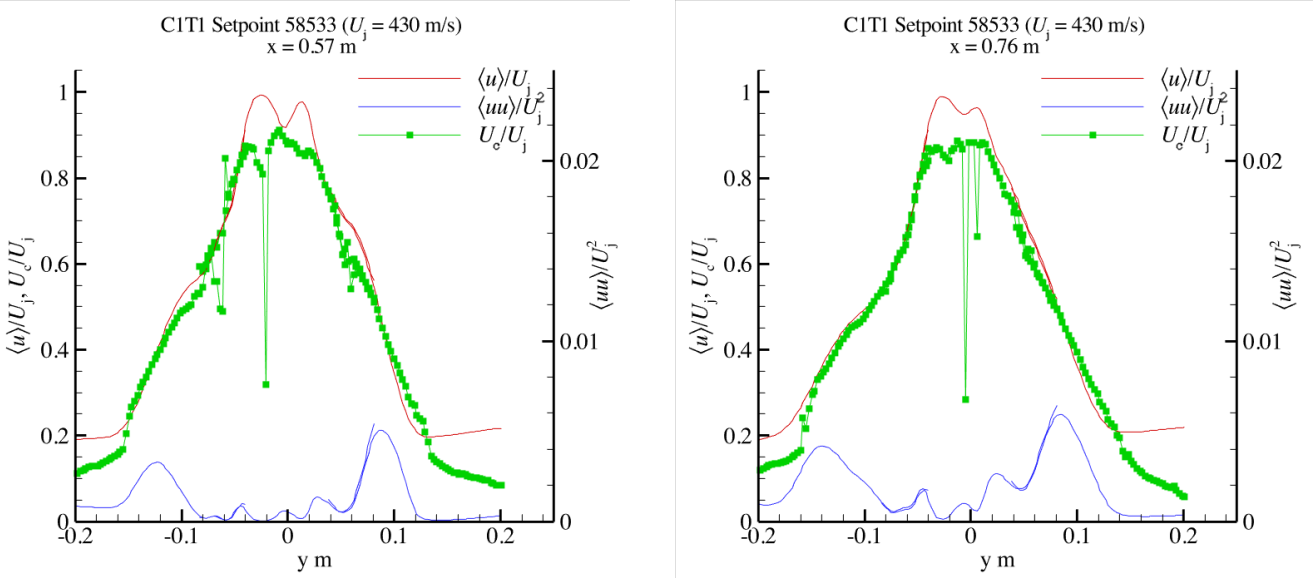

(c)

(d)
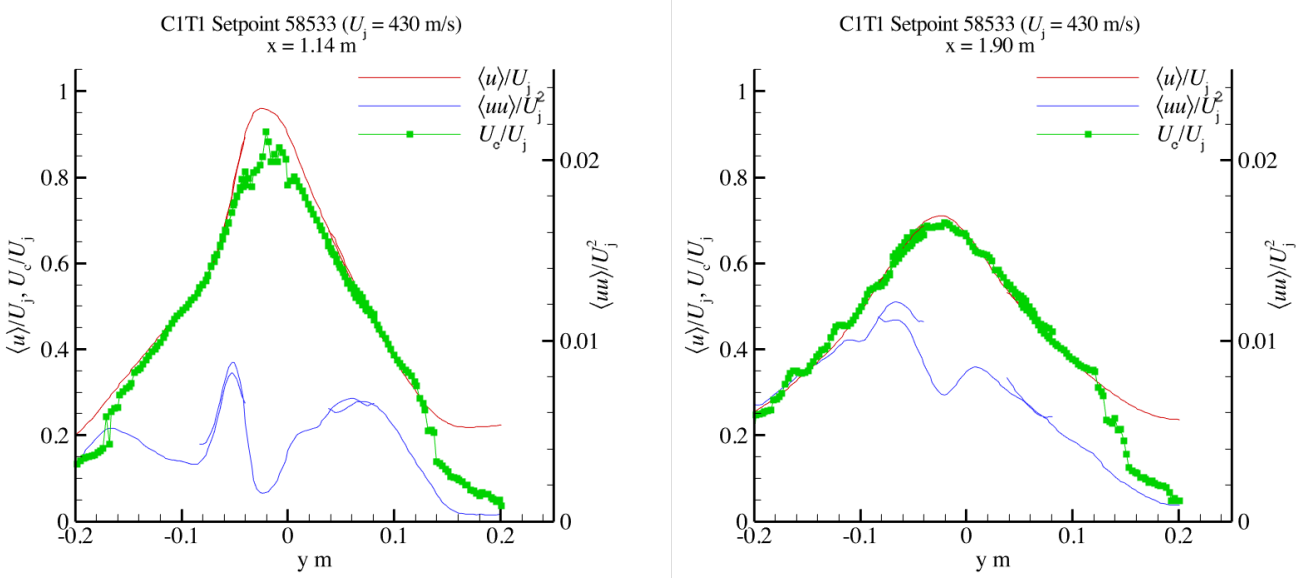

(e)

(f)

Figure 18 Transverse profiles of mean velocity, variance of turbulent velocity, and convection speed for eight axial stations. Jet is dual-stream plume from nozzle C1T1SDCT at setpoint 58533. 

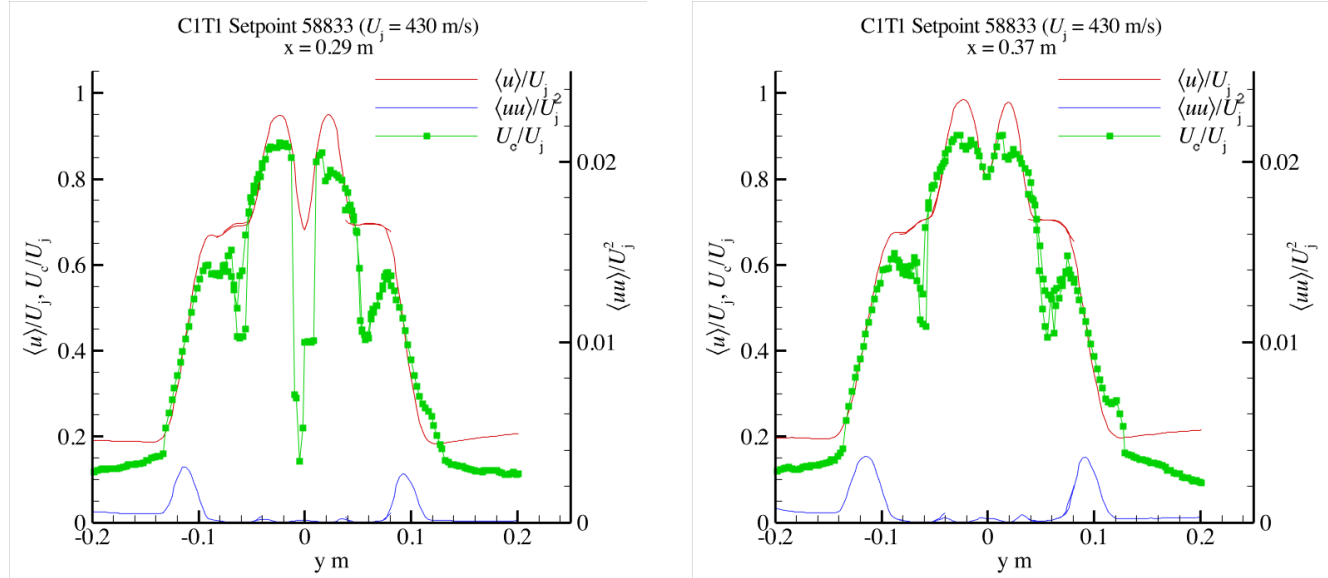

(a)

(b)
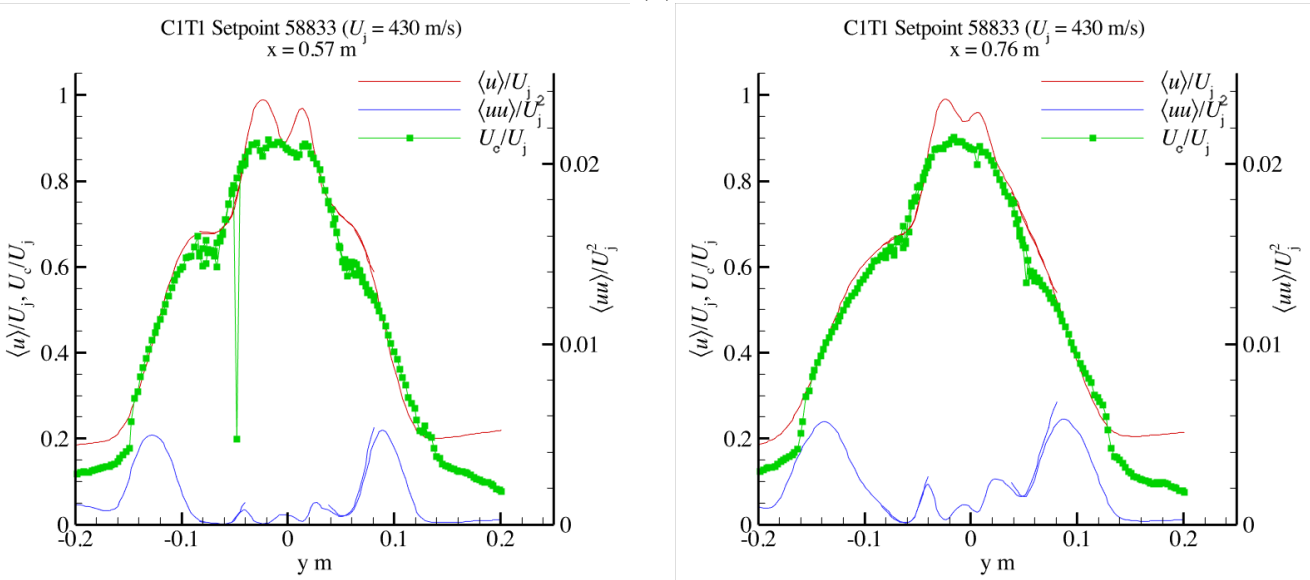

(c)

(d)
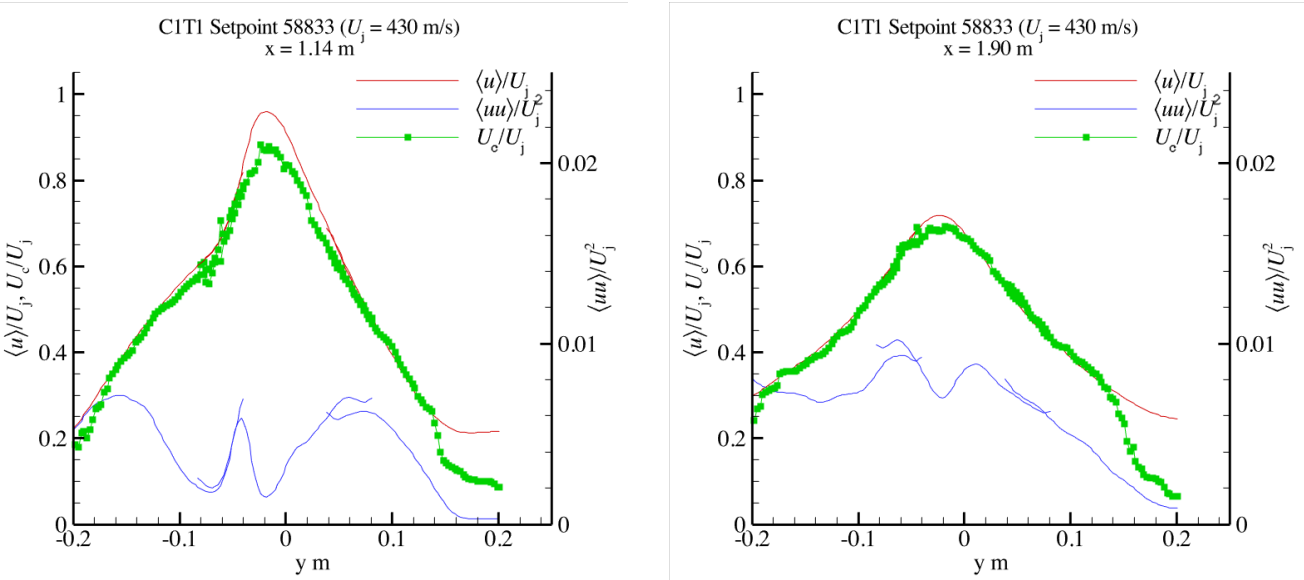

(e)

(f)

Figure 19 Transverse profiles of mean velocity, variance of turbulent velocity, and convection speed for eight axial stations. Jet is dual-stream plume from nozzle C1T1SDCT at setpoint 58833. 

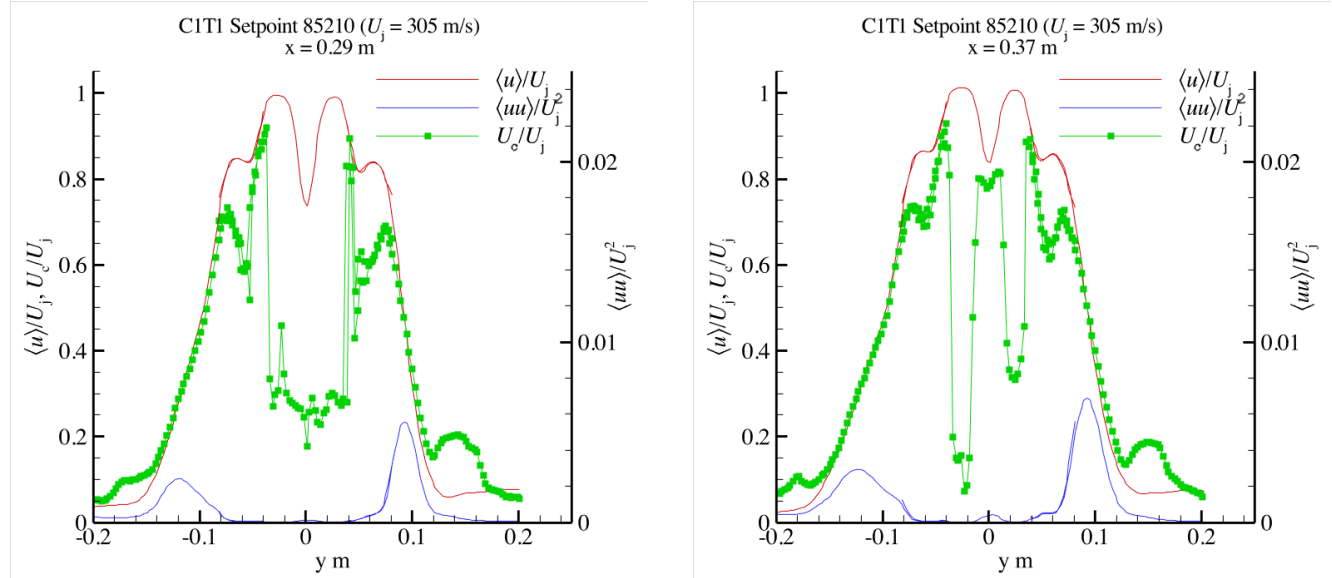

(a)

(b)
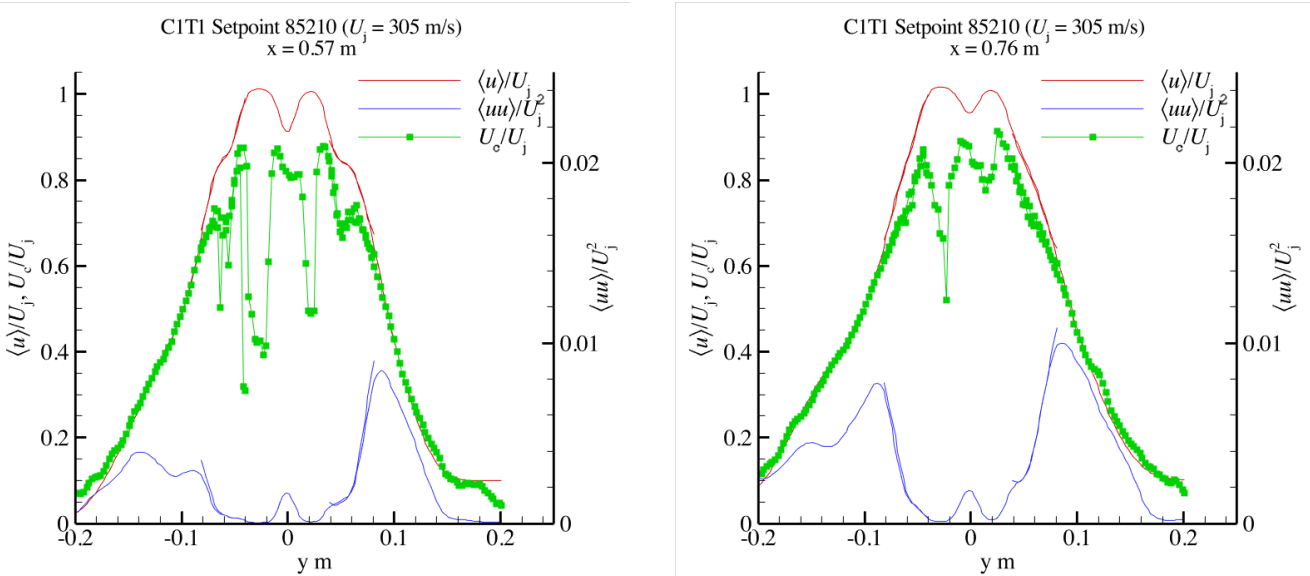

(c)

(d)
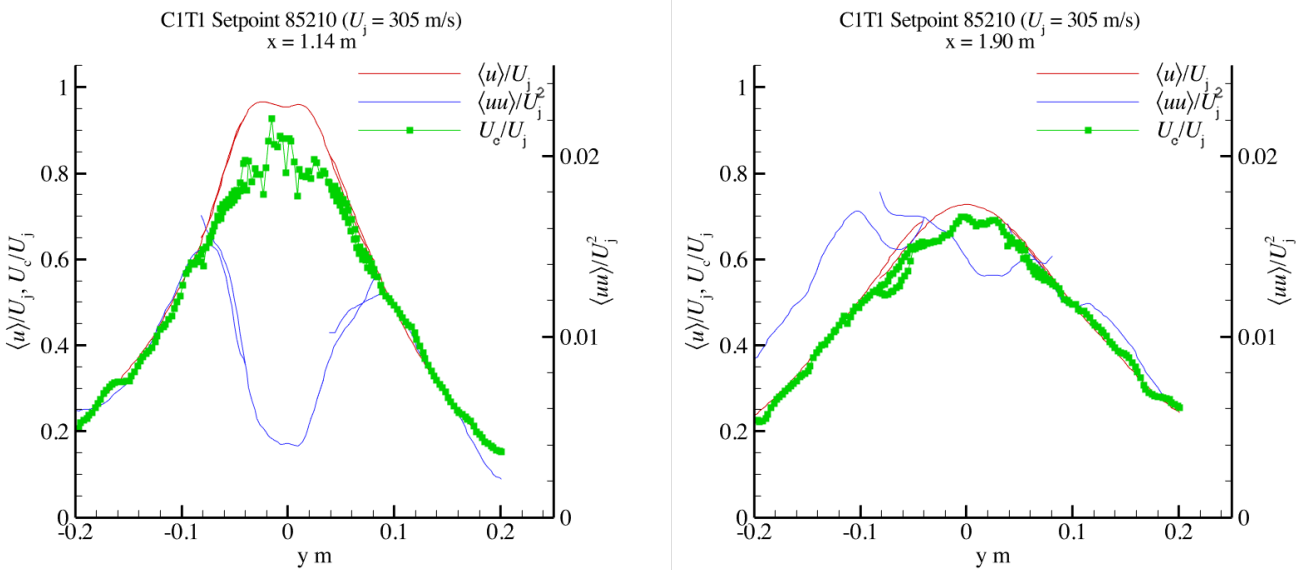

(e)

(f)

Figure 20 Transverse profiles of mean velocity, variance of turbulent velocity, and convection speed for eight axial stations. Jet is dual-stream plume from nozzle C1T1PART at setpoint 58210. 

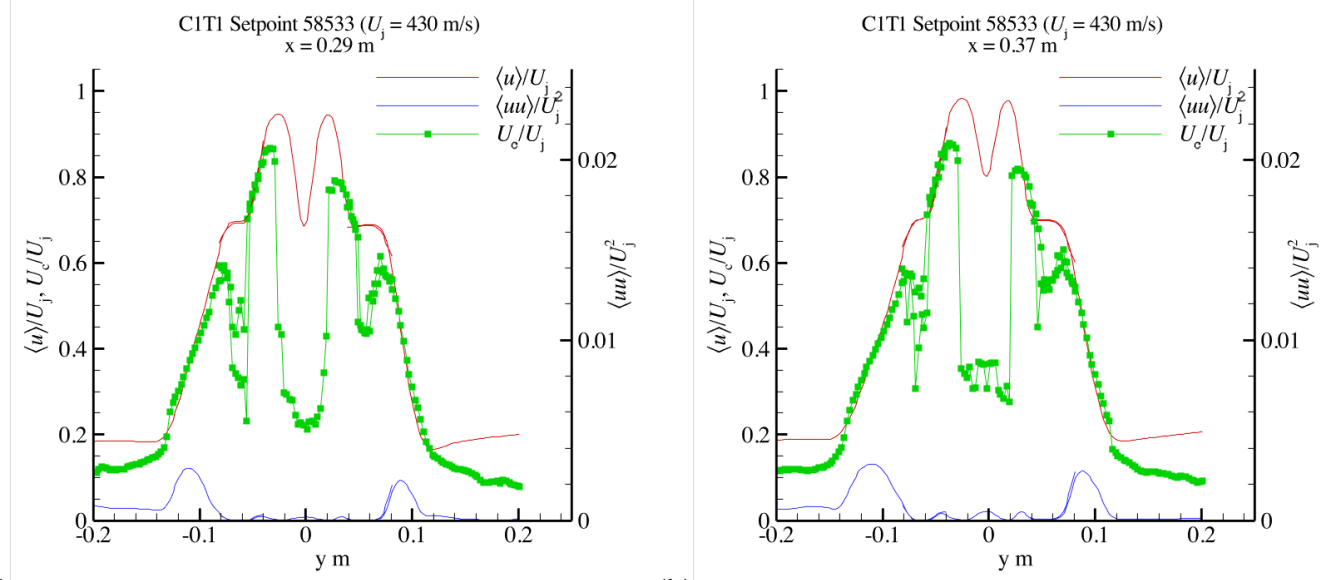

(a)

(b)
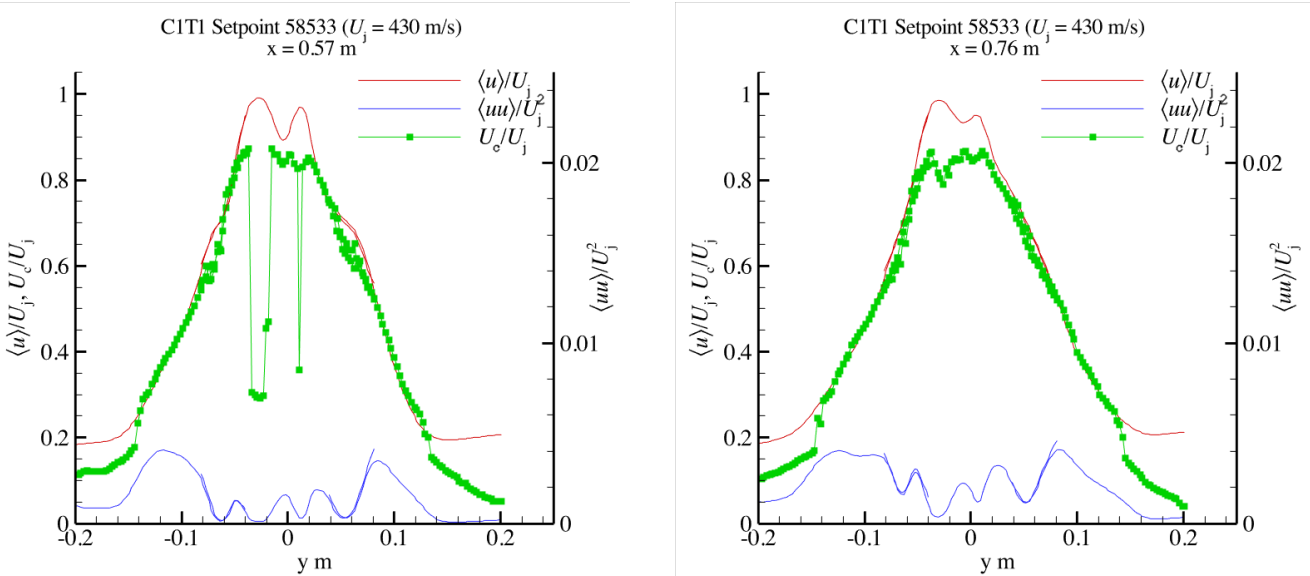

(c)

(d)
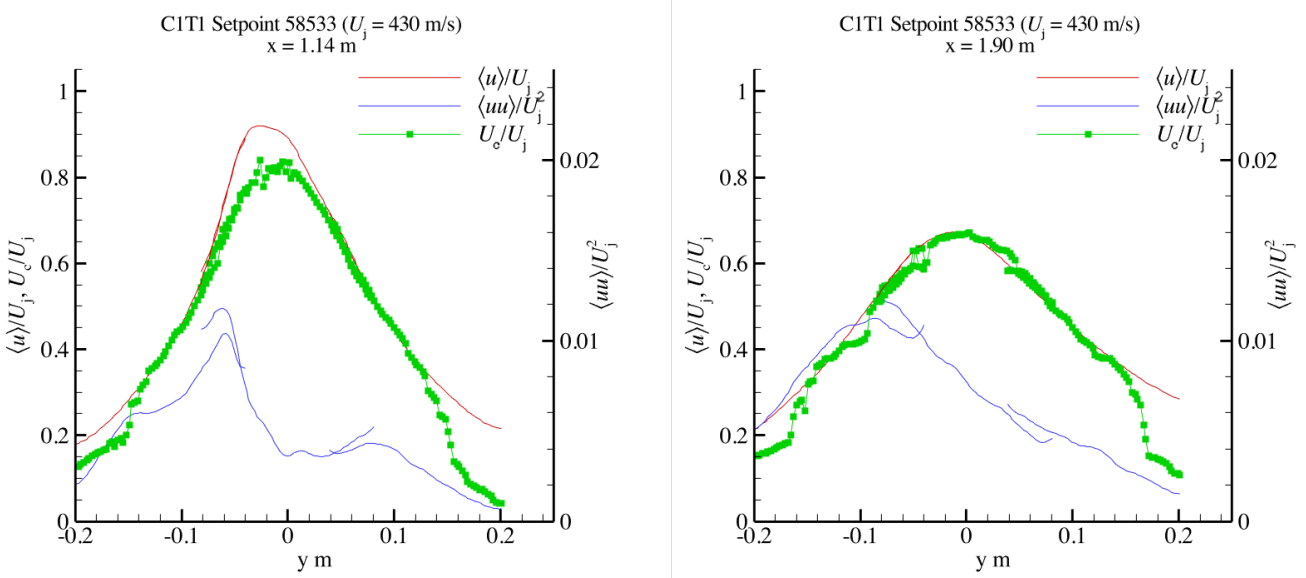

(e)

(f)

Figure 21 Transverse profiles of mean velocity, variance of turbulent velocity, and convection speed for eight axial stations. Jet is dual-stream plume from nozzle C1T1PART at setpoint 58533. 


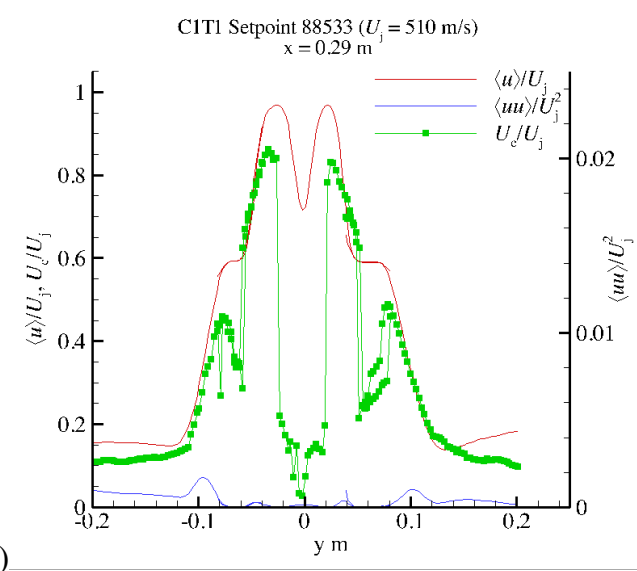

(a)

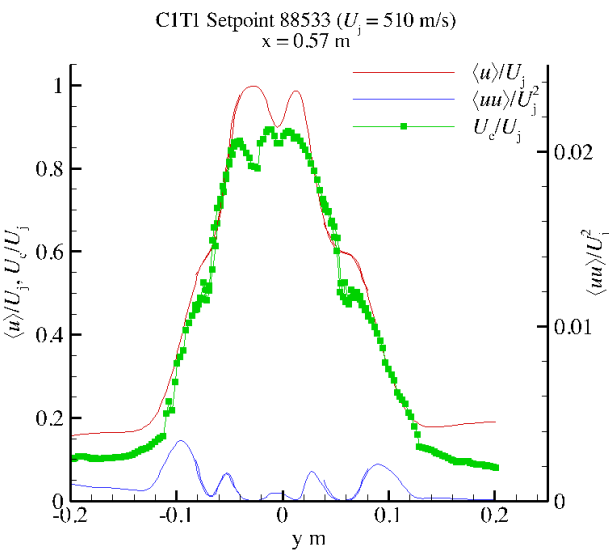

(c)

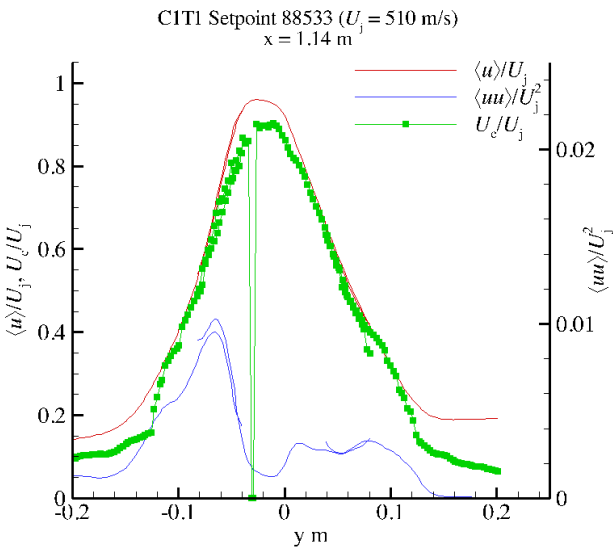

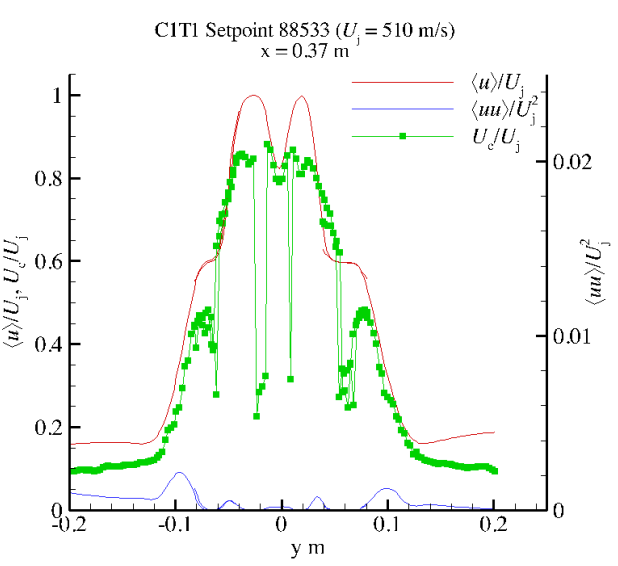

(b)

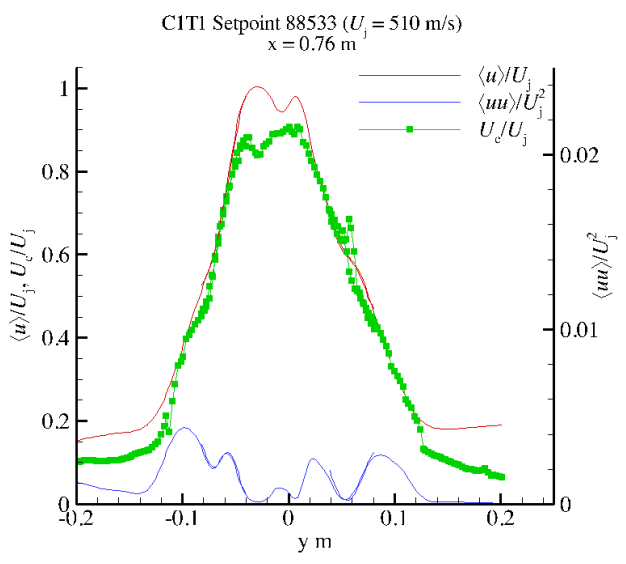

(d)

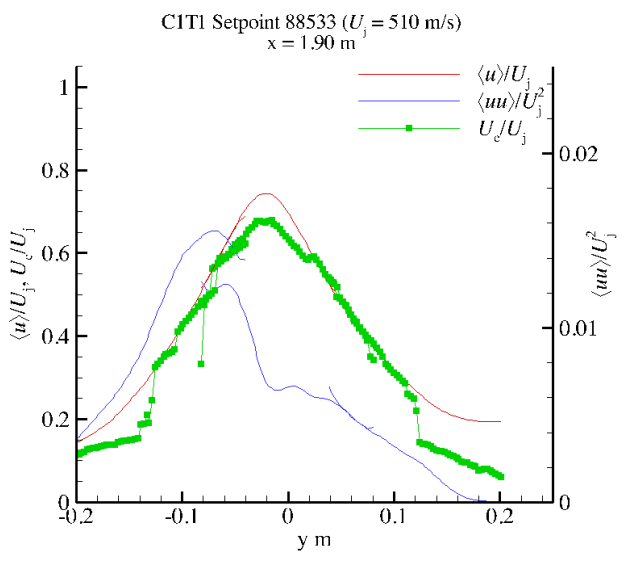

Figure 22 Transverse profiles of mean velocity, variance of turbulent velocity, and convection speed for eight axial stations. Jet is dual-stream plume from nozzle C1T1PART at setpoint 88533.

\section{Discussion}

Looking over all the profiles it is clear that, in general, the convection velocity matched the local mean velocity. Where it did not, the local turbulence was relatively low and the convection velocity was pulled more towards the value of the nearby region with significant turbulent energy. This explains why classical measurements in the potential core of jets found a convection profile that passed through the value of the highest shear and was higher in speed than the mean velocity on the low speed side of the jet and vice versa.

Returning to the original goal of modeling convection speed for engineering applications involving turbulence, it seems that assuming the local convection speed matches the local mean velocity is relatively safe. In regions where 
this is not true it is because the energetic eddies in nearby regions with strong turbulence are dominating the measurement of convection speed of the very small unsteadiness in the flow through their imposed perturbations. In these cases the turbulence being convected may be too small to impact the application. The one place where this is clearly not a good assumption is in applications involving the convection speed of hydrodynamic perturbations, not turbulent eddies, at the jet edge. The PIV method cannot measure such small displacements, and the measurements produced above probably should not be considered relevant for the hydrodynamic perturbations.

Remembering the global observation that convection velocities measured in a profile are influenced by the convection velocity of the most energetic turbulence nearby, it is not surprising that this dynamic might produce convection velocities representative of the outermost shear layer to be felt outside the jet plume. This would agree with the observation by Reference [5] that it is the outer shear layer that drives the near-jet hydrodynamic field because it is the outer shear layer that possesses the most turbulent energy. Nozzle modifications that put the most turbulent energy at the lowest mean velocity would be the best strategy for noise reduction if it is indeed the convection speed of the near-jet hydrodynamic wave packets that drives the large-scale noise of the jet.

\section{Summary}

Profiles of turbulent convection speeds in multi-stream jet plumes were measured in a unique application of time-resolved particle image velocimetry. Starting with unheated single-stream jets where prior data exists for validation, the method was successively applied to two and three-stream plumes, with and without flight stream, and then to cases where the outermost streams were intentionally asymmetric. Profiles of mean velocity, turbulent velocity, and turbulent convection speed were measured at axial stations within and downstream of the potential cores of the jets, and were compared. Convection speed was reliably measured where the turbulence was non-zero, although speeds of small perturbations were often measured in other regions such as the potential core and the ambient.

Away from the initial shear layer, in regions where turbulence existed across the entire plume, the convection speed nearly matched the local mean velocity. In the initial shear layer a convection speed was measured across the jet profile, even where little or no turbulence existed. In keeping with the first observation, this was likely because the turbulent shear layer imposed a perturbation upon the potential flow. With the turbulence peaking at roughly the location where the mean velocity is 0.6 of the centerline velocity, the impression is that this is the convection speed of turbulence across the jet. However, for most applications where convection speed of turbulent energy is required, choosing to model the convection speed as being the local mean velocity is probably best.

Multiple-stream plumes were investigated, which because of their more complicated shear layer stuctures provided many cases to apply this idea. The overall concept that local convection speeds are influenced by those of the most energetic parts of the cross-section was found throughout the data. Regions with little turbulence produced convection speeds that either had high uncertainty or matched that of nearby turbulence. Applying this principle to the problem of jet noise, where high convection velocities in the near-jet hydrodynamic field lead to higher noise production, it is clear that tailoring plumes to produce peak turbulence levels at regions of lower velocity, and hence imposing lower convection velocities on the hydrodynamic field, should yield quieter jets.

\section{Acknowledgments}

The Commercial Supersonics Technology Project under the NASA Advanced Air Vehicles Program supported this work. Many thanks to the staff of the Aero-Acoustic Propulsion Laboratory for their creative hard work carrying out the researcher's crazy ideas. The nozzles used in this test were created by Brenda Henderson and the authors appreciate being able to use them for this work. And thanks to Cliff Brown and Amy Fagan for their helpful corrections of the manuscript.

\section{References}

\footnotetext{
${ }^{1}$ Papamoschou, D, "New method for jet noise reduction in turbofan engines." AIAA Journal 42.11 (2004): 2245 2253.

${ }^{2}$ Papamoschou, D. and Rostamimonjezi, S., "Modeling of Noise Reduction for Turbulent Jets with Induced Asymmetry," AIAA Paper 2012-2158, June 2012.

${ }^{3}$ Reba, R., Narayanan, S., and Colonius, T. "Wave-packet models for large-scale mixing noise." International Journal of Aeroacoustics 9.4-5 (2010): 533-557.
} 
${ }^{4}$ Jordan, P., and Colonius, T. "Wave packets and turbulent jet noise." Annual review of fluid mechanics 45 (2013): 173-195.

${ }^{5}$ Papamoschou, D. and Phong, V. "The Very Near Pressure Field of Single- and Multi-Stream Jets," AIAA Paper 2017-0230, 55th AIAA Aerospace Sciences Meeting, 09 January 2017.

${ }^{6}$ Morris, P.J. and Zaman, K.B.M.Q., "Velocity Measurements in Jets with Application to Noise Source Modeling," AIAA 2009-17 47th AIAA Aerospace Sciences Meeting, 05 January 2009.

${ }^{7}$ Kerherv 'e, F., Jordan, P., Gervais, Y., and Vali ere, J.-C. "Aerodynamic Characterisation of a Supersonic Jet using Two-point Laser Doppler Velocimetry," AIAA 2003-3215, 12-14 May 2003.

${ }^{8}$ Bridges, J, \& Wernet, M.P., "Measurements Of The Aeroacoustic Sound Source In Hot Jets," AIAA paper 2003 3130, May 2003.

${ }^{9}$ Bridges, J. and Wernet, M.P. "Effect of temperature on jet velocity spectra." AIAA Paper 2007-3628, May 2007.

${ }^{10}$ Henderson, B.S. and Leib, S.J. "Three-stream jet noise measurements and predictions." The Journal of the Acoustical Society of America 136.4 (2014): 2080-2080.

${ }^{11}$ Henderson, B.S., and Wernet, M.P. "Characterization of Three-Stream Jet Flow Fields." 54th AIAA Aerospace Sciences Meeting. 2016.

${ }^{12}$ Papamoschou, D., Phong, V., Xiong, J., and Liu, F., "Quiet Nozzle Concepts for Three-Stream Jets", AIAA 2016-0523, 54th AIAA Aerospace Sciences Meeting, January 2016.

${ }^{13}$ Bridges, J. "Simple Scaling Of Multi-Stream Jet Plumes For Aeroacoustic Modeling." AIAA Paper 2016-1637 (2016).

${ }^{14}$ Ecker, T., Ng, W., Lowe, K.T., Henderson, B.S., \& Leib, S.J. "Velocity Statistics and Spectra in Three-Stream Jets." AIAA 2016-1633, (2016).

${ }^{15}$ Bridges, J. and Wernet, M.P. "The NASA subsonic jet particle image velocimetry (PIV) dataset." NASA/TM2011-216807 (2011).

${ }^{16}$ Bridges, J. and Wernet, M.P. "Effect of temperature on jet velocity spectra." AIAA paper 3628 (2007): 2007.

${ }^{17}$ Wernet, M.P. "Time-Resolved PIV for Space-Time Correlations in Hot Jets." 2007 22nd International Congress on Instrumentation in Aerospace Simulation Facilities. IEEE, 2007.

${ }^{18}$ Wernet, M.P., and Hadley, J.A.. "A high temperature seeding technique for particle image velocimetry." Measurement Science and Technology 27.12 (2016): 125201.

${ }^{19}$ Birch, Stanley, et al. "Deflected Fan Flows: Nozzle Installation Effects." AIAA Paper 2010-3922 (2010).

${ }^{20}$ Birch, S., Khritov, K., Maslov, V., Mironov, A., \& Secundov, A. “An experimental study of flow asymmetry in co-axial jets," AIAA 2005-2845 (2005). 